\title{
Optimizing large-scale structure data analysis with the theoretical error likelihood
}

\author{
Anton Chudaykin, ${ }^{1,2, *}$ Mikhail M. Ivanov $\odot,{ }^{3,2, \dagger}$ and Marko Simonović ${ }^{4, \#}$ \\ ${ }^{1}$ Department of Physics \& Astronomy, McMaster University, \\ 1280 Main Street West, Hamilton, Ontario L8S 4M1, Canada \\ ${ }^{2}$ Institute for Nuclear Research of the Russian Academy of Sciences, \\ 60th October Anniversary Prospect, 7a, 117312 Moscow, Russia \\ ${ }^{3}$ Center for Cosmology and Particle Physics, Department of Physics, \\ New York University, New York, New York 10003, USA \\ ${ }^{4}$ Theoretical Physics Department, CERN, 1 Esplanade des Particules, Geneva 23 CH-1211, Switzerland
}

(Received 2 October 2020; accepted 22 January 2021; published 16 February 2021)

\begin{abstract}
An important aspect of large-scale structure data analysis is the presence of non-negligible theoretical uncertainties, which become increasingly important on small scales. We show how to incorporate these uncertainties in realistic power spectrum likelihoods by an appropriate change of the fitting model and the covariance matrix. The inclusion of the theoretical error has several advantages over the standard practice of using the sharp momentum cut $k_{\max }$. First, the theoretical error covariance gradually suppresses the information from the short scales as the employed theoretical model becomes less reliable. This allows one to avoid laborious measurements of $k_{\max }$, which is an essential part of the standard methods. Second, the theoretical error likelihood gives unbiased constraints with reliable error bars that are not artificially shrunk due to overfitting. In realistic settings, the theoretical error likelihood yields essentially the same parameter constraints as the standard analysis with an appropriately selected $k_{\max }$, thereby effectively optimizing the choice of $k_{\max }$. We demonstrate these points using the large-volume $N$-body data for the clustering of matter and galaxies in real and redshift space. In passing, we validate the effective field theory description of the redshift space distortions and show that the use of the one-parameter phenomenological Gaussian damping model for fingers-of-God causes significant biases in parameter recovery.
\end{abstract}

DOI: $10.1103 /$ PhysRevD.103.043525

\section{INTRODUCTION}

Galaxy clustering on large scales becomes ever more important in modern cosmology. The measurements of baryon acoustic oscillations (BAO) and the power spectrum shape in the current data allow one to determine cosmological parameters with precision that rivals the cosmic microwave background analysis [1-7]. Even more progress is expected in the era of the upcoming high-precision surveys such as Euclid [8,9] and DESI [10]; see e.g., [11-14].

One of the crucial ingredients for measurement of cosmological parameters from a large-scale structure (LSS) is an accurate covariance matrix for a given summary statistic.

\footnotetext{
*chudy@ms2.inr.ac.ru

†mi1271@nyu.edu

¥marko.simonovic@cern.ch
}

Published by the American Physical Society under the terms of the Creative Commons Attribution 4.0 International license. Further distribution of this work must maintain attribution to the author(s) and the published article's title, journal citation, and DOI.
The question of how to estimate covariance matrices and how they impact cosmological constraints has stimulated a broad line of research over the past decades. This includes perturbative calculations [15-20], measurements from mock catalogs (e.g., [21-23]), and studying the systematic biases that can arise due to the uncertainties in the covariance matrix [24-27]. While these significant efforts focus mainly on statistical errors, it is important to note that there is another aspect of the covariance matrix treatment that has attracted attention only recently. This is the theoretical error (TE) covariance, which is as important as the statistical covariance for any realistic large-scale structure analysis [11,12,28,29]. The TE covariance originates from imperfect knowledge of the theory model that is used to fit the data. The LSS observables are sensitive to various nonlinear effects whose accurate description is quite challenging, both in analytical approaches to LSS clustering and in $N$-body or hydrodynamical simulations. The importance of these nonlinear effects grows on small scales and therefore impacts a large number of Fourier modes that are important to constrain cosmological parameters. 
Theoretical error becomes a leading source of uncertainty when the statistical errors become sufficiently small (either due to a large volume of a survey or the analysis being pushed to smaller scales), at which point it has to be included in the analysis to avoid biasing the output cosmology. The standard approach to deal with this situation is to assume that the fitted theory model is perfect up to a certain scale (e.g., $k_{\max }$ in the power spectrum case) and perform the analysis with this data cut. This approach has a number of disadvantages. First, it is very time consuming, since the only way to determine the optimal $k_{\max }$ is to run many Markov-chain Monte Carlo (MCMC) analyses of the realistic mock data samples; see e.g., [1,30-33]. Moreover, if a different model or a set of priors is used, one has to revalidate the $k_{\max }$ choice because the parameter variances and degeneracy orientations change in this case $[30,34]$. The second drawback of the $k_{\max }$ analysis is that biases for different parameters become sizable at very different scales. This makes the choice of $k_{\max }$ ambiguous. The usual approach is to choose the cuts such that biases on all cosmological parameters are significantly smaller than the statistical error. On the one hand, this choice is the most conservative as it ignores possible improvements on cosmological parameters that are unbiased for larger $k_{\max }$. On the other hand, it still does not guarantee the absence of bias in nuisance parameters, which can be problematic when doing a joint analysis of different observables (for example, power spectrum and bispectrum) or combining different datasets. Finally, using a sharp data cut neglects two important properties of the power spectrum: (a) the broadband power spectrum is smooth, and (b) the shape of the common features, such as BAO wiggles, can be reliably calculated for any wave number [35-41]. ${ }^{1}$ These two facts reveal yet another important limitation of the standard $k_{\max }$ analysis: it neglects all information from scales beyond $k_{\max }$, a part of which can be recovered assuming some reasonable smoothening of the power spectrum and using the shape information from the BAO wiggles.

In this paper we show how all these issues can be resolved including the theoretical error covariance in the analysis. As a first step, we rederive the theoretical error covariance emphasizing the similarity of this procedure with the exact marginalization over a nuisance parameter. This example naturally suggests that the theoretical error likelihood should include both the mean and the covariance of the theoretical uncertainty, with identical shapes. Then, we propose a new way of extracting these quantities directly from the mock data and argue that this method gives a more reliable estimate of theoretical error than the original approach based exclusively on perturbation theory.

We apply the TE formalism to the $N$-body simulation data with volume $\sim 100(\mathrm{Gpc} / h)^{3}$, similar to the

\footnotetext{
${ }^{1}$ This statement can be generalized to the primordial oscillating features in the power spectrum and bispectrum $[42,43]$.
}

cumulative volume of upcoming spectroscopic galaxy surveys. We explicitly illustrate how the TE approach allows one to obtain accurate and optimal constraints that are independent of the choice of $k_{\max }$ and that are not affected by overfitting. Moreover, the TE guarantees that both the principal components and their one-dimensional (1D) projections onto the particular parameter planes are unbiased, along with the variances of these parameters. Finally, we show that using the TE covariance indeed improves constraints on cosmological parameters by including extra information from the $\mathrm{BAO}$ wiggles and exploiting the smoothness of the broadband power spectrum. ${ }^{2}$ It is important to stress that all these results can be obtained with a single MCMC analysis, in contrast with the standard $k_{\max }$ approach.

We scrutinize the effect of TE covariance on the power spectrum analyses in four difference setups: dark matter and galaxies in real and redshift space. We analyze the large-volume $N$-body simulation data using the power spectra calculated in the framework of the effective field theory of a large-scale structure (see [1,30] and references therein) and explicitly demonstrate that the true input cosmology is extracted in an unbiased manner in all of these different examples. Our analyses also validate the implementation of the effective field theory for various tracers with the CLASS-PT code [44]. In this context, our work can be viewed as a companion paper of Ref. [44], as it proves that the accuracy of CLASS-PT meets the requirements of future high-precision surveys.

The paper is organized as follows. We give a theoretical background on the theoretical error in Sec. II. Section III specifies our methodology, the theoretical model, and $N$ body simulations. Then, we present the TE analysis and confront it with the standard $k_{\max }$ approach for dark matter in real space in Sec. IV, dark matter in redshift space in Sec. V, galaxies in real space in Sec. VI, and galaxies in redshift space in Sec. VII. We conclude in Sec. VIII. Some additional material is presented in the Appendixes. Some details on the choice of the theoretical error are given in Appendix A. Some details on the fingers-of-God modeling for dark matter in redshift space are given in Appendix B. Finally, Appendix C presents tests of the stability of our constraints with respect to the choice of fiducial cosmology used to calibrate the theoretical error.

\section{THEORETICAL ERROR LIKELIHOOD}

In this section we review the theoretical error formalism. Let us first repeat the derivation of Ref. [28] focusing on the power spectrum likelihood,

\footnotetext{
${ }^{2}$ This approach has recently been validated in a slightly different context of the reanalysis of the BAO data from the BOSS survey in Ref. [5].
} 


$$
-2 \ln \mathcal{L}=C_{i j}^{-1}\left(P_{i}^{\mathrm{th}}-P_{i}^{\mathrm{d}}\right)\left(P_{j}^{\mathrm{th}}-P_{j}^{\mathrm{d}}\right),
$$

where the sum over $(i, j)$ is implicitly assumed, $C_{i j}$ is the data covariance matrix, $P^{\text {th }}$ is the true theoretical model, and $P^{\mathrm{d}}$ is the data vector. In practice, the true theoretical model is not known and we use an approximate model $P_{i}^{\text {approx }}$, e.g., the one-loop perturbation theory, which becomes less and less accurate on small scales. Theoretical error is the difference between the (unknown) true theory and this approximation,

$$
P_{i}^{\mathrm{TE}} \equiv P_{i}^{\mathrm{th}}-P_{i}^{\mathrm{approx}}
$$

Let us assume that $P_{i}^{\mathrm{TE}}$ is drawn from a Gaussian distribution with mean $\bar{P}_{i}^{\mathrm{TE}}$ and the covariance $C^{(\mathrm{E})}$,

$$
\mathcal{P}\left(P^{\mathrm{TE}}\right) \propto \exp \left\{-\frac{1}{2}\left(C^{(E)}\right)_{i j}^{-1}\left(P_{i}^{\mathrm{TE}}-\bar{P}_{i}^{\mathrm{TE}}\right)\left(P_{j}^{\mathrm{TE}}-\bar{P}_{j}^{\mathrm{TE}}\right)\right\} .
$$

Marginalizing our original likelihood (2.1) over the theoretical error we get the following marginalized likelihood:

$$
\begin{aligned}
-2 \ln \mathcal{L}_{\mathrm{marg}}= & \left(C^{(\mathrm{tot})}\right)_{i j}^{-1}\left(P_{i}^{\mathrm{approx}}+\bar{P}_{i}^{\mathrm{TE}}-P_{i}^{\mathrm{d}}\right) \\
& \times\left(P_{j}^{\mathrm{approx}}+\bar{P}_{j}^{\mathrm{TE}}-P_{j}^{\mathrm{d}}\right), \\
C^{(\mathrm{tot})}= & C+C^{(\mathrm{E})}
\end{aligned}
$$

It is instructive to consider a few illustrative examples. First, let us assume that the theoretical error covariance is diagonal, $C_{i j}^{(\mathrm{E})} \propto E_{i}^{2} \delta_{i j}$, the mean $\bar{P}_{i}^{\mathrm{TE}}=0$, and

$$
E_{i}=\left\{\begin{array}{ll}
\infty, & \text { if } k \geq k_{\max } \\
0, & \text { otherwise }
\end{array} .\right.
$$

With this choice the bins with $k \geq k_{\max }$ are thrown away from the likelihood and the bins with $k<k_{\max }$ are assumed to have no theoretical error. This limit corresponds to the standard analysis with fixed $k_{\max }$.

\section{A. Theoretical error from analytic nuisance parameter marginalization}

Let us now take a look at the opposite extreme, where the theoretical error is correlated in all $k$ bins. A simple example is given by

$$
P_{i}^{\mathrm{TE}}=\alpha\left(\frac{k_{i}}{k_{\mathrm{NL}}}\right)^{4} P_{\mathrm{lin}}\left(k_{i}\right) \equiv \alpha \mathcal{E}_{i},
$$

whose coefficient is expected to be Gaussian distributed as

$$
\alpha \sim \mathcal{N}\left(\bar{\alpha}, \sigma_{\alpha}^{2}\right)
$$

Such a form of the TE as well as the prior on $\alpha$ can be motivated either from perturbation theory (as higher derivative counterterms) or from $\mathrm{N}$-body simulations. Let us now marginalize over $\alpha$ just as one normally marginalizes over nuisance parameters in realistic cosmological analyses. The marginalized Gaussian likelihood can easily be obtained from (2.1),

$$
\begin{aligned}
-2 \ln \mathcal{L}_{\text {marg }}^{\alpha}= & \left(C^{(\text {tot })}\right)_{i j}^{-1}\left(P_{i}^{\text {approx }}+\bar{\alpha} \mathcal{E}_{i}-P_{i}^{\mathrm{d}}\right) \\
& \times\left(P_{j}^{\text {approx }}+\bar{\alpha} \mathcal{E}_{j}-P_{j}^{\mathrm{d}}\right), \\
C_{i j}^{(\mathrm{tot})}= & C_{i j}+\sigma_{\alpha}^{2} \mathcal{E}_{i} \mathcal{E}_{j},
\end{aligned}
$$

where we have used the Sherman-Morrison identity. We can see that in this case, since the $k$ dependence of the TE is entirely fixed, the new covariance is fully correlated even if the data covariance $C_{i j}$ is diagonal. We stress that so far our calculation has been exact; i.e., up to marginalization over $\alpha$, the likelihood (2.8) contains the same information as the original likelihood (2.1).

\section{B. Theoretical error model}

In reality, the exact $k$ dependence of the theoretical error is not known (otherwise it would be included in the theory model), but we want to marginalize over it just as we did with $\alpha$. In other words, we want to marginalize over all possible curves within some bounds given by the expected size of the theoretical error and with sufficient degree of smoothness. This can be achieved choosing an appropriate TE covariance matrix. Following [28], we use the ansatz

$$
C_{i j}^{(\mathrm{E})}=E_{i} E_{j} e^{-\frac{\left(k_{i}-k_{j}\right)^{2}}{2 \Delta k^{2}}},
$$

where we introduced finite coherence scale $\Delta k$, whereas $E_{i}$ is some $k$-dependent smooth envelope for the theoretical error. Note that we neglect the cosmology dependence of $C^{(E)}$ in the same way as it is customarily done for the data covariance [45] (see [46] for the justification of this practice for the Cosmic Microwave Background (CMB) ). The coherence scale ensures that the neighboring bins at a distance smaller than $\Delta k$ are almost fully correlated, but the allowed theoretical uncertainties can freely vary only on separations larger than $\Delta k$. Choosing the appropriate limits, we can recover the two previous examples. In the limit $\Delta k \rightarrow \infty$ all $k$ bins are correlated and the theoretical error corresponds to adding the shape $E(k)$ to the theory model and marginalizing over its amplitude. In the opposite limit $\Delta k \rightarrow 0$ all bins can fluctuate independently and the theoretical error becomes diagonal as in our first example. Note that this limit is unphysical because it allows theoretical uncertainty to have arbitrarily fast oscillations and it makes the result dependent on the binning.

We will proceed with the following choice for the coherence scale 


$$
\Delta k=0.1 h / \mathrm{Mpc} .
$$

On the one hand, it is small enough to allow for typical variations of nonlinear corrections within the usual range of scales used in the data analysis. On the other hand, it is big enough to ensure that the broadband theoretical error is correlated on scales corresponding to the frequency of the $\mathrm{BAO}$ wiggles, $k_{\mathrm{BAO}} \sim 0.01 \mathrm{~h} / \mathrm{Mpc}$. This can help extract the information from the $\mathrm{BAO}$ even if the envelope $E_{i}$ is large.

Indeed, the theoretical error with this coherence length will effectively discard the broadband information, but will retain the oscillatory features such as the BAO wiggles [5]. Therefore, while increasing the total covariance, the theoretical error can possibly improve the constraints on cosmological parameters by taking into account the information on the smoothness of $E_{i}$ and exploiting any additional features.

Once the coherence length is fixed, the envelope and the mean of the theoretical error have to be chosen as well. This is the most difficult task, since it strongly depends on the exact model being used and the observable being analyzed. In the context of perturbation theory a reasonable guess is $\bar{P}_{i}=0$ and the envelope can be estimated as the typical size of higher loop corrections not included in the model [28]. This theory-inspired estimate does not use any input from simulations or data, but, strictly speaking, it is expected to be accurate only on the order-of-magnitude basis. Indeed, we will see that the perturbation theory-inspired theoretical error can overestimate the actual uncertainty in real space by a factor of few. The perturbation theory estimate is even less reliable in redshift space and can significantly underestimate the true theoretical uncertainty, particularly for higher order multipoles. We illustrate this issue in detail in Appendix A.

In order to avoid making some overly optimistic or pessimistic choices in our analyses, we choose an alternative, simulation-driven strategy to estimate the mean and the envelope of the theoretical error. The main idea is to estimate the TE covariance from the difference between the data and the best-fit model inferred from an analysis based on large scales. We assume that the typical size of the envelope is equal to the TE mean,

$$
E_{i}=\bar{P}_{i}^{(\mathrm{TE})},
$$

in which case the theoretical error is fully characterized by a single shape $\bar{P}^{(\mathrm{TE})}(k)$. Equation (2.11) is motivated by the example of the counterterm marginalization (2.8), where the mean and the variance of the theoretical error have identical shapes.

\section{Practical realization}

In practice, we suggest the following concrete algorithm to estimate the theoretical error and construct the likelihood:
1. Choose some fiducial cosmological model. Given the real of mock $N$-body data, we compute the theory prediction for cosmological parameters fixed to some fiducial values. This step is similar, in spirit, to choosing a fiducial model for the statistical covariance matrix.

2. Select some fiducial data cut $k_{\max }^{\mathrm{fid}}$. This data cut should be reasonably small, such that the theoretical error is negligible for this data cut. We use the following procedure. Having fixed some fiducial cosmology in the first step, we minimize the usual power spectrum likelihoods with respect to nuisance parameters for a set of $k_{\max }$ and plot the best-fit reduced $\chi^{2}$ statistic as a function of $k_{\max }$. The corresponding profiles are flat up to a certain scale $k_{2-\text { loop}}$, at which they start exhibiting significant scale dependence. This is the scale at which the twoloop corrections become important. Any choice of $k_{\max }^{\text {fid }}$ that is lower than $k_{2-\text { loop }}$ is suitable for our purposes. In practice, we use $k_{\max }^{\mathrm{fid}}=k_{2-\text { loop }}-\Delta k^{\prime}$, where $\Delta k^{\prime}=0.04 h \mathrm{Mpc}^{-1}$. We have tested our procedure by varying $k_{\max }^{\mathrm{fid}}$ and found that it does not have any significant effect on the results. The details about the choice of the data cuts $k_{\max }^{\mathrm{fid}}$ can be found in Appendix A.

3. Obtain a fiducial theoretical spectrum at $k_{\max }^{\mathrm{fid}}$. To that end we fit the power spectrum data varying only the nuisance parameters at our fiducial $k_{\max }^{\mathrm{fid}}$. This analysis is very fast, since it requires computing a single best-fit point involving only the nuisance parameters. As a result we obtain the best-fit theoretical curve $P^{\text {best-fit }}(k)$.

4. Take the best-fit theory curve and compute the theoretical curve envelope:

$$
P_{i}^{(\mathrm{TE})}=P_{i}^{\mathrm{d}}-P^{\text {best-fit }}\left(k_{i}\right) .
$$

This curve may have some stochastic scatter induced by the data vector $P_{i}^{\mathrm{d}}$. To remove it, one can fit $P_{i}^{(\mathrm{TE})}$ with a smooth polynomial. We will call this smooth curve $\bar{P}_{i}^{(\mathrm{TE})}$.

5. Construct the TE likelihood using $\bar{P}_{i}^{(\mathrm{TE})}$ as follows:

$$
\begin{aligned}
-2 \ln \mathcal{L}(P(\vec{\theta}))= & \left(C+C^{(\mathrm{E})}\right)_{i j}^{-1}\left(P(\vec{\theta})+\bar{P}_{i}^{(\mathrm{TE})}-P_{i}^{\mathrm{d}}\right) \\
& \times\left(P(\vec{\theta})+\bar{P}_{i}^{(\mathrm{TE})}-P_{j}^{\mathrm{d}}\right), \quad(2.13)
\end{aligned}
$$

where $\vec{\theta}$ is the vector of cosmological parameters that we want to fit and

$$
C_{i j}^{(\mathrm{E})}=\bar{P}_{i}^{(\mathrm{TE})} \bar{P}_{j}^{(\mathrm{TE})} e^{-\frac{\left(k_{i}-k_{j}\right)^{2}}{2 \Delta k^{2}}}, \quad \text { with } \quad k=0.1 \mathrm{~h} / \mathrm{Mpc} .
$$

An important comment is in order. The key ingredient of our algorithm is the theoretical error envelope that depends 
on the three particular choices: the fiducial data cut $k_{\max }^{\mathrm{fid}}$, the fiducial cosmological model, and the mock (or real) data points. In principle, one has to verify that the final result is stable with respect to these three choices. This can be done by iterating the fiducial cosmology to match the best-fit output spectra, ${ }^{3}$ and by varying $k_{\max }^{\text {fid }}$ and simulated data points. However, all these three choices are correlated and effectively they have the same result-the change of the theoretical error envelope. Hence, essentially one only needs to verify that the final results do not vary much as the theoretical error envelope is changed within some reasonable range. We have performed these consistency checks focusing on the variation of the fiducial cosmology and $k_{\max }^{\mathrm{fid}}$. This test has shown that constraints on some cosmological parameters are affected by the choice of the theoretical error envelope in the unrealistic cases of pure dark matter clustering, while the realistic redshift space galaxy power spectrum results are fully consistent and stable with respect to the variation of the theoretical error envelope.

Finally, it is worth pointing out that the most robust strategy is to calibrate the theoretical error from simulated mock catalogs of a given survey and use it in all analyses of the real data. Unlike the statistical uncertainty, the theoretical error covariance does not scale with volume or shot noise and hence needs to be calibrated only once for a particular tracer and redshift bin. Besides, in this case one can include the scatter produced by variations of $N$-body simulation parameters in the theoretical error budget, which is required in order to ensure the stability of the final cosmological constraints.

\section{The choice of theoretical cross-covariance for redshift space multipoles}

So far all our formulas were written for the simplest case of the real space power spectrum analysis. The treatment of the theoretical error becomes slightly more complicated in redshift space. In the plane-parallel (flat sky) approximation, the redshift space power spectrum is the function of two variables, the wave number $k$ and the cosine between the wave vector $\mathbf{k}$ and the unit line-of-sight direction $\hat{\mathbf{z}}$, denoted by $\mu$,

$$
\mu \equiv(\mathbf{k} \cdot \hat{\mathbf{z}}) / k
$$

The redshift space power spectrum can be conveniently cast in the form of Legendre multipoles $L_{\ell}$,

$$
\begin{aligned}
P(k, \mu) & =\sum_{\ell=0} P_{\ell}(k) L_{\ell}(\mu), \\
P_{\ell} & \equiv \frac{2 \ell+1}{2} \int_{-1}^{1} d \mu L_{\ell}(\mu) P(k, \mu),
\end{aligned}
$$

\footnotetext{
${ }^{3}$ In the context of the statistical error, this is a common practice in photometric and spectrosopic surveys; see [47] and references therein.
}

where $\ell=0,2,4$ are monopole, quadrupole, and hexadecapole moments, respectively.

We need to implement the condition that the theoretical error is a smooth function in both $k$ and $\mu$. If the theoretical error curve is a single fixed function of $k$ and $\mu$, and only its amplitude is unknown, an explicit marginalization over this amplitude [similar to Eq. (2.8)] suggests that the theoretical error covariance is fully correlated across $\mu$ and $k$ bins. At the level of the Legendre multipoles, this means that the theoretical error multipoles are $100 \%$ correlated. However, since the precise shape of the theoretical error is not known by definition, we will impose some finite correlation between the TE multipoles using the model similar to that describing the correlation of $k$ bins,

$$
C_{i j}^{(\mathrm{E})\left(\ell \ell^{\prime}\right)}=E_{i}^{(\ell)} E_{j}^{\left(\ell^{\prime}\right)} e^{-\frac{\left(k_{i}-k_{j}\right)^{2}}{2 \Delta k^{2}}} e^{-\frac{\left(\ell-\ell^{\prime}\right)^{2}}{2 \Delta \ell^{2}}}
$$

where $i, j$ now run only over the $k$ bins. In what follows, we will use the minimal nontrivial multipole coherence length $\Delta \ell=2$.

The appearance of $e^{-\frac{\left(\ell-\epsilon^{\prime}\right)^{2}}{2 \Delta \ell^{2}}}$ in Eq. (2.17) can be understood from the following argument. If the theoretical error in $(k, \mu)$ space were characterized by a single parameter, i.e., in analogy with Eq. (2.6) it would be given by

$$
P^{(\mathrm{TE})}(k, \mu)=\alpha\left(\frac{k}{k_{\mathrm{NL}}}\right)^{4} \mu^{4} P_{\mathrm{lin}}(k)\left(b_{1}+f \mu^{2}\right)^{2},
$$

then the theoretical error covariance between the multipoles, obtained after marginalization over $\alpha$, would be $100 \%$ correlated. The shape (2.18) indeed appears at the next-to-leading order (NLO) in the Taylor expansion of the redshift space mapping [1]. However, if the theoretical error were characterized by an independent parameter for every single multipole $\left(L_{\ell}\right.$ is the Legendre polynomial of order $\ell)$, i.e.,

$P^{(\mathrm{TE})}(k, \mu)=\alpha_{0} \mathcal{E}_{0}\left(k_{i}\right) L_{0}(\mu)+\alpha_{2} \mathcal{E}_{2}\left(k_{i}\right) L_{2}(\mu)+\cdots$,

then marginalizations over the nuisance parameters $\alpha_{\ell}$ would produce the effective theoretical error covariance that is diagonal in multipole numbers. Thus, having a finite coherence length in the multipole space represents a sensible compromise between these two extreme situations.

\section{METHODOLOGY, SIMULATIONS, AND COVARIANCES}

In this section we discuss technical details of our analysis: the simulation data, the theoretical model, and the covariance matrices. 


\section{A. Theoretical model}

We use one-loop cosmological perturbation theory to compute power spectra for dark matter and galaxies in real and redshift spaces. The details of our theoretical model can be found in Ref. [44]; it includes IR resummation to capture the nonlinear evolution of the BAO wiggles $[35-40,48]$ and UV counterterms that are required to account for the effects of short-scale dynamics, whose description is impossible within perturbation theory itself [49-51].

\section{B. Data}

Throughout the paper we will use a suite of LasDamas Oriana simulations [52], which consists of 40 boxes with $L=2.4 \mathrm{Gpc} / h$ on a side, totaling the volume of $553(\mathrm{Gpc} / h)^{3}$. The cosmological parameters used to generate mock catalogs are $h=0.7, \Omega_{m}=0.25, \Omega_{b}=0.04$, $\sigma_{8}=0.8\left(A_{s}=2.22 \times 10^{-9}\right), n_{s}=1$, and $\sum m_{\nu}=0$. The details of LasDamas simulation can be found [54]. To reduce the stochastic scatter, we fit the mean of power spectra extracted from 40 independent simulation boxes. However, the statistical error corresponding to the total simulation volume is so small that the two-loop corrections and inaccuracies of $\mathrm{N}$-body modeling can supersede cosmic variance already on very large scales. In order to be more realistic, we will use the covariance that corresponds to $V=100(\mathrm{Gpc} / h)^{3}$ and not to the actual LasDamas volume. This reduced volume is comparable to the total volume of future spectroscopic surveys such as DESI [10] or Euclid [11], which allows one to interpret our results in the context of these surveys.

We will analyze the statistics of dark matter particles and galaxies in real and redshift space. The redshift snapshots available to us are $z=0$ and $z=0.974$ for dark matter, and $z=0.342$ for galaxies. The galaxy distribution is generated using the HOD model that matches the luminous red galaxy (LRG) sample of the BOSS survey [55], with the shot noise level

$$
\bar{n}^{-1}=1.05906 \times 10^{4}[\mathrm{Mpc} / h]^{3} .
$$

The theoretical nonlinear spectra are generated with the CLASS-PT code [44] that computes one-loop perturbation theory integrals using the FFTLog method [56]. We run our MCMC chains with the MontePython sampler [57,58], analyze these chains, and produce triangle plots with the GetDist package [59]. In all analyses of this paper, we fit the following set of cosmological parameters:

$$
\left\{\omega_{\mathrm{cdm}}, h, A^{1 / 2}, n_{s}\right\}, \quad A \equiv A_{s} / A_{s, \text { fid }},
$$

and also present the derived parameters $\Omega_{m}$ and $\sigma_{8}$. We fix $\omega_{b}$ to the fiducial value used in simulations. This is done in order

\footnotetext{
${ }^{4}$ We also use the CMB monopole temperature $T_{0}=2.725 \mathrm{~K}$, which is required to set various normalizations in CLASS [53].
}

to simulate the $\omega_{b}$ prior that can be taken from Big Bang Nucleosynthesis or Planck in a realistic analysis [1].

\section{Statistical covariance}

We use the covariance matrix in the Gaussian approximation to describe sample variance [15]. This approximation is very accurate for the power spectrum at least for scales $k_{\max } \leq 0.4 h \mathrm{Mpc}^{-1}$ [47]. The Gaussian covariance for the real space power spectrum takes the following form for the matter-matter, galaxy-galaxy power spectra $\left(P_{\mathrm{mm}}\right.$ and $\left.P_{\mathrm{gg}}\right)$ and galaxy-matter cross-spectrum $\left(P_{\mathrm{gm}}\right)$, respectively,

$$
\begin{aligned}
C\left[P_{\mathrm{mm}}(k), P_{\mathrm{mm}}\left(k^{\prime}\right)\right]= & \frac{2}{N_{k}} P_{\mathrm{mm}}^{2}(z, k) \delta_{k k^{\prime}}, \\
C\left[P_{\mathrm{gg}}(k), P_{\mathrm{gg}}\left(k^{\prime}\right)\right]= & \frac{2}{N_{k}}\left(P_{\mathrm{gg}}(z, k)+\frac{1}{\bar{n}(z)}\right)^{2} \delta_{k k^{\prime}}, \\
C\left[P_{\mathrm{gm}}(k), P_{\mathrm{gm}}\left(k^{\prime}\right)\right]= & \frac{2}{N_{k}}\left(P_{\mathrm{gg}}(z, k)+\frac{1}{2 \bar{n}(z)}\right) \\
& \times P_{\mathrm{mm}}(z, k) \delta_{k k^{\prime}},
\end{aligned}
$$

where $N_{k}=V 4 \pi k^{2} d k /(2 \pi)^{3}$ is the number of modes in a $k$ bin of size ( $d k=0.0025 \mathrm{~h} / \mathrm{Mpc}$ in all our analyses) and $\delta_{k k^{\prime}}$ is the Kronecker delta. In practice, we use the measurements $P_{\mathrm{mm}}$ and $P_{\mathrm{gm}}$ in the covariance matrix estimates (3.3).

The Gaussian covariance between the redshift space galaxy multipoles is given by [11]

$$
\begin{aligned}
C_{k_{i} k_{j}}^{\left(\ell_{1} \ell_{2}\right)}= & \frac{2}{N_{k}}\left(2 \ell_{1}+1\right)\left(2 \ell_{2}+1\right) \int_{0}^{1} d \mu L_{\ell_{1}}(\mu) L_{\ell_{2}}(\mu) \\
& \times\left[P\left(k_{i}, \mu\right)+\frac{1}{\bar{n}}\right]^{2} \delta_{i j} .
\end{aligned}
$$

Expressions (3.3) and (3.4) are valid for both dark matter and galaxy statistics. It is worth noting that the galaxy power spectrum should be considered along with shot noise term (3.1) which accounts for the discrete nature of galaxies. In practice, we evaluate (3.4) in the linear (Kaiser) approximation [60].

All analyses for sharp data cuts are performed with the usual statistical covariances. To run the analyses with the theoretical error, we supplement the statistical covariances with the theoretical ones, (2.9) in real space, and (2.17) in redshift space.

\section{DARK MATTER IN REAL SPACE}

We start by analyzing the dark matter power spectrum in real space. To that end we use the one-loop IR-resummed template of [38] with the addition of the effective dark matter sound speed counterterm $\gamma[49,50]$, which will be the only nuisance parameter in our analysis,

$$
P=P_{\text {tree }}+P_{1-\text { loop }}^{\mathrm{SPT}}-2 \gamma k^{2} P_{\text {tree }}(k),
$$



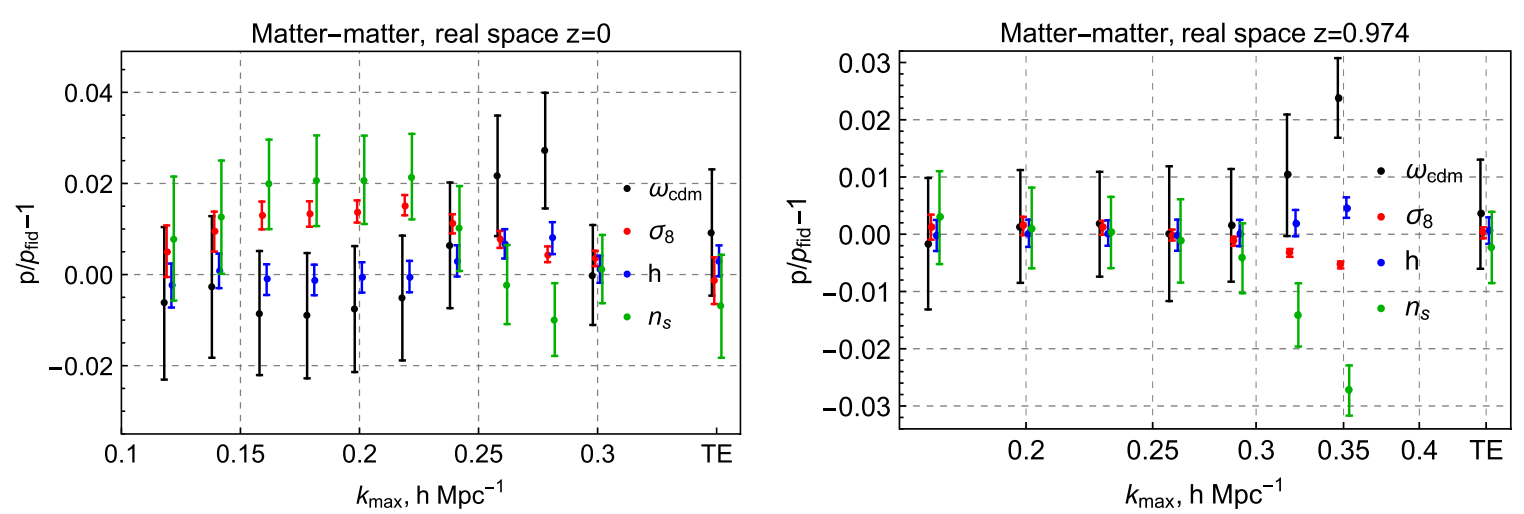

FIG. 1. Marginalized 1D limits for cosmological parameters from the matter power spectrum at $z=0$ (left panel) and $z=0.974$ (right panel) as a function of $k_{\max }$. The rightmost group of points in each panel corresponds to the TE analysis. All parameters are normalized to their fiducial values.

where $P_{1-\text { loop }}^{\mathrm{SPT}}$ is the one-loop correction computed in standard perturbation theory (SPT) [61].

As a first step, we fit the data vectors with sharp cuts $k_{\max }$ and pure statistical covariance. As a second step, we add the theoretical error to the analysis. The results in this case do not depend on $k_{\max }$ provided that it is reasonably high. In practice, we cut the data vector at $k=k_{\mathrm{NL}} \approx 0.3 h / \mathrm{Mpc}$ and $0.5 \mathrm{~h} / \mathrm{Mpc}$ for $z=0$ and $z=0.974$, respectively. The results for the marginalized $1 \mathrm{D}$ variances of cosmological parameters as functions of $k_{\max }$ are shown in Fig. 1. Note that we have run a complete MCMC analysis for every $k_{\max }$ in this plot. The rightmost points correspond to the theoretical error analysis. Let us begin with the $z=0$ case. One can see from the left panel of Fig. 1 that the measured optimal parameters start deviating from the fiducial ones at $k_{\max }=0.12 \mathrm{~h} / \mathrm{Mpc}$. But starting from $k_{\max }=0.24 \mathrm{~h} / \mathrm{Mpc}$, the estimates start moving in the opposite direction and accidentally become fully compatible with the fiducial cosmology at $k_{\max }=0.3 \mathrm{~h} / \mathrm{Mpc}$.
Clearly, this behavior is caused by overfitting and the constraints at $k_{\max }=0.3 \mathrm{~h} / \mathrm{Mpc}$ cannot be trusted even though they enclose the fiducial values within $1 \sigma$. Importantly, the error bars obtained at $k_{\max }=0.3 \mathrm{~h} / \mathrm{Mpc}$ are much smaller than the ones we got before the fit started deviating from the truth at $k_{\max }=0.12 \mathrm{~h} / \mathrm{Mpc}$. This illustrates the danger of the $k_{\max }$ approach, which can be significantly affected by overfitting. In what follows, we choose $k_{\max }=0.12$ to be a baseline cut at $z=0$ as the estimated parameters match the fiducial cosmology within $1 \sigma$ there, but $\sigma_{8}$ becomes biased by more than $2 \sigma$ for larger $k_{\max }$.

Now we focus on the $z=0.974$ case, whose results are shown in the right panel of Fig. 1 . We see that $\sigma_{8}$ undergoes an excursion beyond $1 \sigma$ when $k_{\max }$ is varied between $0.14 \mathrm{hMpc}^{-1}$ and $0.3 \mathrm{hMpc}^{-1}$, but accidentally crosses the fiducial value at $k_{\max }=0.28 \mathrm{hMpc}^{-1}$. The error bars at $k_{\max }=0.14 \mathrm{hMpc}^{-1}$ and $k_{\max }=0.28 \mathrm{hMpc}^{-1}$ are significantly different, but both measurements enclose the fiducial

TABLE I. The marginalized 1D intervals for the cosmological parameters estimated from the Las Damas real space dark matter power spectra at $z=0$ and $z=0.974$. The table contains fitted parameters (first column), fiducial values used in simulations (second column), and the results for two different redshifts: $z=0$ (third and fourth columns) and $z=0.974$ (fifth and sixth columns). In either case we display the results of the baseline $k_{\max }$ analysis and the outcome of the theoretical error approach. $\gamma$ is quoted in units $\left[h^{-1} \mathrm{Mpc}\right]^{2}$.

\begin{tabular}{|c|c|c|c|c|c|}
\hline \multirow[b]{2}{*}{ Par } & \multirow[b]{2}{*}{ Fid } & \multicolumn{2}{|c|}{$z=0$} & \multicolumn{2}{|c|}{$z=0.974$} \\
\hline & & $k_{\max }=0.12$ & $\mathrm{TE}$ & $k_{\max }=0.28$ & $\mathrm{TE}$ \\
\hline$\omega_{\mathrm{cdm}}$ & 0.1029 & $0.1023_{-1.8 \times 10^{-3}}^{+1.6 \times 10^{-3}}$ & $0.1039_{-1.5 \times 10^{-3}}^{+1.4 \times 10^{-3}}$ & $0.1033_{-1.1 \times 10^{-3}}^{+1.0 \times 10^{-3}}$ & $0.1033_{-1.0 \times 10^{-3}}^{+9.4 \times 10^{-4}}$ \\
\hline$h$ & 0.7 & $0.6983_{-3.4 \times 10^{-3}}^{+3.3 \times 10^{-3}}$ & $0.7021_{-2.4 \times 10^{-3}}^{+2.4 \times 10^{-3}}$ & $0.7004_{-1.8 \times 10^{-3}}^{+1.6 \times 10^{-3}}$ & $0.7005_{-1.7 \times 10^{-3}}^{+1.6 \times 10^{-3}}$ \\
\hline$n_{s}$ & 1 & $1.008_{-0.013}^{+0.014}$ & $0.9930_{-0.011}^{+0.011}$ & $0.9955_{-6.1 \times 10^{-3}}^{+6.4 \times 10^{-3}}$ & $0.9977_{-6.1 \times 10^{-3}}^{+6.3 \times 10^{-3}}$ \\
\hline$A$ & 1 & $1.014_{-0.020}^{+0.020}$ & $0.9897_{-0.016}^{+0.016}$ & $0.9972_{-9.4 \times 10^{-3}}^{+9.7 \times 10^{-3}}$ & $0.9977_{-9.0 \times 10^{-3}}^{+.9 .4 \times 10^{-3}}$ \\
\hline$\Omega_{m}$ & 0.25 & $0.2499_{-2.3 \times 10^{-3}}^{+2.2 \times 10^{-3}}$ & $0.2504_{-1.7 \times 10^{-3}}^{+1.6 \times 10^{-3}}$ & $0.2504_{-1.2 \times 10^{-3}}^{+1.2 \times 10^{-3}}$ & $0.2504_{-1.2 \times 10^{-3}}^{+1.1 \times 10^{-3}}$ \\
\hline$\sigma_{8}$ & 0.8003 & $0.8044_{-4.5 \times 10^{-3}}^{+4.6 \times 10^{-3}}$ & $0.7992_{-4.1 \times 10^{-3}}^{+4.1 \times 10^{-3}}$ & $0.7996_{-6.8 \times 10^{-4}}^{+6.8 \times 10^{-4}}$ & $0.8005_{-7.7 \times 10^{-3}}^{+7.8 \times 10^{-4}}$ \\
\hline$\gamma$ & $\ldots$ & $1.60_{-0.31}^{+0.33}$ & $1.19_{-0.32}^{+0.33}$ & $0.498_{-0.024}^{+0.025}$ & $0.535_{-0.029}^{+0.029}$ \\
\hline
\end{tabular}


cosmology within $1 \sigma$; hence the results depend on whether one wants to be more conservative or aggressive. In practice, the second option is often more popular, and thus we choose $k_{\max }=0.28 \mathrm{~h} / \mathrm{Mpc}$ as a baseline data cut at $z=0.974$

Let us compare the baseline $k_{\max }$ results with the theoretical error analysis, focusing first on the $z=0$ case. The values of the marginalized $1 \mathrm{D}$ variances are displayed in Table I. The marginalized 2D triangle plot for the $k_{\max }=$ $0.12 \mathrm{~h} / \mathrm{Mpc}$ and the theoretical error (TE) analysis are displayed in Fig. 2. The theoretical error envelope for TE analysis was computed using a best-fit model at fiducial $k_{\max }^{\mathrm{fid}}=0.10 h / \mathrm{Mpc}$ (see Appendix A for more detail). We see that the TE covariance helps us measure all cosmological parameters without any significant bias. Moreover, there is a moderate improvement over the $k_{\max }$ analysis for all cosmological parameters of interest: the errors on $\omega_{\mathrm{cdm}}$, $h, n_{s}$, and $\sigma_{8}$ reduce by $20 \%, 30 \%, 20 \%$, and $10 \%$, respectively.

It is important to test whether our results depend on the shape of the theoretical error envelope. The details on this test can be found in Appendix C. Choosing a different fiducial cosmology to compute the theoretical error template, we have found that the constraints on $\omega_{\text {cdm }}$ and $h$ do not change, whereas the posteriors for $n_{s}$ and $\sigma_{8}$ shrink by $10 \%$ and $25 \%$, respectively. While these changes are very marginal, they suggest that the improvements that we have obtained for $n_{s}$ and $\sigma_{8}$ should be taken with a grain of salt and in conjunction with our choice of the theoretical error envelope.

Now we turn to the $z=0.974$ case, which is more relevant for upcoming surveys. The values of the

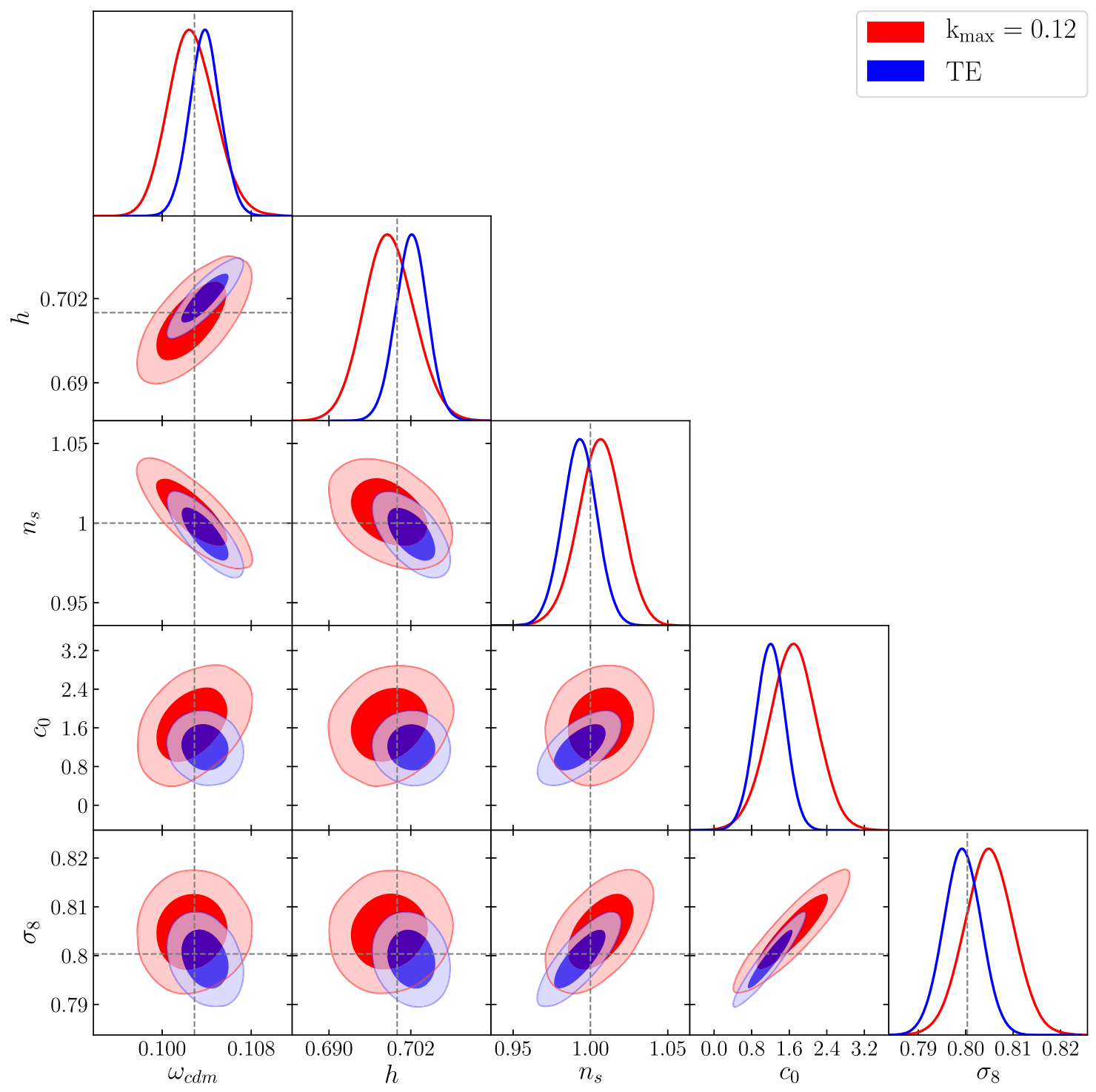

FIG. 2. Triangle plot for the cosmological and nuisance parameters measured from the real space dark matter power spectrum of the Las Damas simulations at $z=0$. 


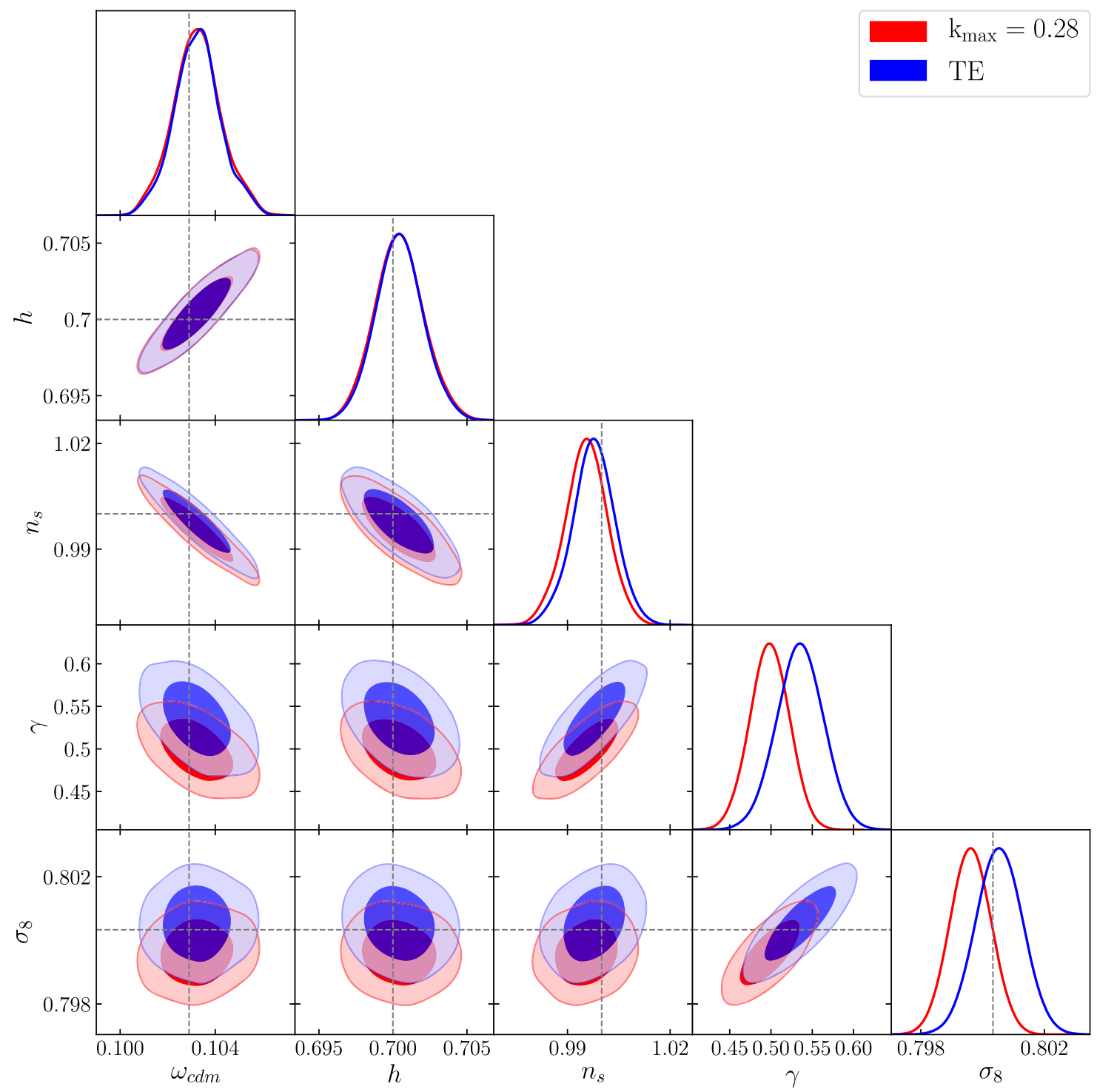

FIG. 3. Triangle plot for the cosmological and nuisance parameters measured from the real space dark matter power spectrum of the Las Damas simulations at $z=0.974$.

marginalized 1D variances for the $k_{\max }=0.28 \mathrm{~h} / \mathrm{Mpc}$ and the TE analysis are displayed in Table I. The envelope for TE analysis was computed with $k_{\max }^{\mathrm{fid}}=0.26 \mathrm{~h} / \mathrm{Mpc}$ (see Appendix A for more detail). We found that using the TE covariance matrix leads to unbiased estimates of all cosmological parameters. However, unlike the $z=0$ case, it does not noticeably improve the error bars compared to the baseline $k_{\max }$ results. This happens mainly because the $\mathrm{BAO}$ wiggles are strongly suppressed for wave numbers larger than $0.28 \mathrm{~h} / \mathrm{Mpc}$ [38]; thus going to larger wave numbers with the TE does not yield as much new information as in the $z=0$ case since the baseline $k_{\max }$ is larger. Moreover, our TE constraints on $\sigma_{8}$ are somewhat weaker (by 13\%) than those from the baseline $k_{\max }$ analysis, and the mean value is not shifted from the true cosmology. From the marginalized 2D triangle plot shown in Fig. 3 one clearly sees the source of this worsening: the degeneracy between $\sigma_{8}$ and the counterterm $\gamma$ is broken less efficiently when the theoretical error is taken into account. Recall that the measurements of the effective sound speed are very sensitive to the two-loop corrections [62]. If we ignore these corrections, the measurement of $\gamma$ will be biased and the error bars will be underestimated. Given the apparent degeneracy $\gamma-\sigma_{8}$, this propagates into the posterior of $\sigma_{8}$, whose width is underestimated too. In contrast, including the theoretical error allows one to marginalize over the two-loop contributions and get a more correct measurement of $\gamma$ and $\sigma_{8}$ with unbiased, albeit larger, error bars. This shows that the TE covariance is imperative in order to get accurate estimates of the parameter variances. 


\section{DARK MATTER IN REDSHIFT SPACE}

In this section we study the clustering of dark matter in redshift space. In a real survey one always observes dark matter tracers (such as galaxies) in redshift space. In this case, it is hard to disentangle between the nonlinear effects of redshift space and the nonlinear galaxy bias. Thus, the case of redshift space dark matter will clearly show us how well perturbation theory can describe nonlinearities induced specifically by redshift space mapping. We will see that the theoretical error plays a crucial role in this analysis too, and allows for noticeable improvement of the cosmological constraints.

Normally the analysis of the redshift space power spectrum is limited to the first three nontrivial moments $\ell=0$, 2, 4 because only these moments exist in linear theory. We have found that the hexadecapole signal is dominated by a systematic leakage from lower moments due to discreteness effects. Given this reason, we perform our analysis only for the monopole and quadrupole moments. We fit the IR-resummed one-loop power spectrum,

$P^{(z)}(k, \mu)=P_{\text {tree }}^{\mathrm{IR}-\text { res }}(k, \mu)+P_{1-\text { loop }}^{\mathrm{SPT}, \mathrm{IR}-\mathrm{res}}(k, \mu)+P_{\text {ctr }}(k, \mu)$,

where $P_{\text {ctr }}$ denotes counterterms which fix the UV dependence of the loops and parametrizes the ignorance about short scale dynamics. This term also addresses the "fingersof-God" effect [63]. In what follows we describe this phenomenon perturbatively along the lines of the effective field theory.

The section has the following outline. First, we validate the effective field theory treatment of fingers-of-God and compare it with popular phenomenological prescriptions in Sec. VA. Then, we present the measurements of cosmological parameters from the mock redshift space power spectrum for the cases of the theoretical error approach and the sharp momentum cuts in Sec. V B.

\section{A. Fingers-of-God modeling}

The biggest challenge in the analytic description of redshift space distortions is the fingers-of-God effect, induced by virialized motions of dark matter particles (or galaxies) in halos. The virialized velocity field is fully nonlinear, and there is very little hope that it can be described analytically from first principles. However, the effect of fingers-of-God on long-wavelength fluctuations can be captured within effective field theory by a finite set of operators. In particular, the lowest order corrections are given by $[64,65]$

$P_{\nabla^{2} \delta}(k, \mu)=-2\left(c_{0}^{\prime}+c_{2}^{\prime} f \mu^{2}+c_{4}^{\prime} f^{2} \mu^{4}\right) k^{2} P_{\text {lin }}(k)$,

where $c_{0}, c_{2}$, and $c_{4}$ are called "counterterms" and $f \equiv$ $d \ln D / d \ln a$ is the logarithmic growth factor. The values and time dependence of counterterms are not known a priori, and hence we treat them as free parameters and marginalize over their amplitudes.

It is quite natural to keep three different free coefficients since they fix the UV dependence of different loop diagrams and capture different physical effects. Namely, the monopole counterterm includes the contribution similar to the higher-derivative bias $\nabla^{2} \delta$ (along with the dark matter effective sound speed operator), which is absent for higher order multipole moments. In contrast, the quadrupole counterterm captures the dispersion of the short-scale velocity field. In what follows, we will justify that keeping independent coefficients in every counterterm is necessary for a proper description of dark matter clustering on large scales.

The expansion (5.2) assumes that the dimensionful coefficients $c_{i}$ are small,

$$
c_{i} k^{2} \ll 1, \quad i=0,2,4 .
$$

However, the peculiar velocities can be rather large, which can violate the condition of the applicability of perturbation theory (5.3) even on large scales. For instance, the BOSS galaxies are expected to have the velocity dispersion $\sim 6 \mathrm{Mpc} / h$, which gives an estimate $c_{2} \sim 50[\mathrm{Mpc} / h]^{2}[1]$. It implies that the characteristic momentum scale of higher order short-scale velocity cumulants is significantly lower than the nonlinear scale that controls gravitational nonlinearities in real space, $k_{\mathrm{NL}} \sim 0.5 h \mathrm{Mpc}^{-1}$ [28]. Hence, the usual one-loop power spectrum models (5.1) and (5.2) can become insufficient for an accurate description of the data even on large scales. We proceed by introducing an additional counterterm to capture the redshift space nonlinearities at next-to-leading order [1],

$$
P_{\nabla_{z}^{4} \delta}=-b_{4}(\mu k f)^{4}\left(b_{1}+f \mu^{2}\right)^{2} P_{\operatorname{lin}}(k),
$$

where $b_{4}$ denotes the next-to-leading counterterm and $b_{1}=1$ for the dark matter. The redshift space mapping effectively generates an expansion in powers of $\nabla_{z}^{2} \delta$. From this point of view, the extra counterterm can be viewed as the $\nabla_{z}^{4} \delta$ contribution in this expansion. We choose the nextto-leading order counterterm (5.4) to be universal for all multipole moments, as dictated by the redshift space mapping. We assume that the contributions from other physical effects (higher-derivative counterterms, etc.) are subdominant since their nonlinear scale is similar to $k_{\mathrm{NL}}$ for the real space dark matter.

It is worth mentioning that in a realistic LSS data analysis the redshift determination errors generate additional corrections to the power spectrum. For example, the redshift smearing of quasars from the eBOSS survey is described by a $k^{2} \mu^{2} P_{\text {lin }}$-like counterterm at leading order in the derivative expansion [66], but a more realistic model requires higher order corrections with at least one additional free parameter to capture deviations from 
Gaussianity [67]. An accurate modeling of redshift errors beyond simplistic one-parameter models will also be important for the Euclud/DESI-type emission line galaxies; see Ref. [41] for an extended discussion. This is another reason why it is important to have free coefficients in front of the various redshift space counterterms: they also capture different systematic effects.

The full counterterm contribution is given by $P_{\text {ctr }}=$ $P_{\nabla^{2} \delta}+P_{\nabla_{z}^{4} \delta}$. Doing the integrals with the Legendre polynomials, we get ${ }^{5}$

$$
\begin{aligned}
P_{\mathrm{ctr}, \ell=0}= & -2 c_{0} k^{2} P_{\operatorname{lin}}(k)-b_{4} f^{4}\left(\frac{f^{2}}{9}+\frac{2 f b_{1}}{7}+\frac{b_{1}^{2}}{5}\right) k^{4} P_{\operatorname{lin}}(k), \\
P_{\mathrm{ctr}, \ell=2}= & -2 c_{2} \frac{2 f}{3} k^{2} P_{\operatorname{lin}}(k)-b_{4} f^{4}\left(\frac{40 f^{2}}{99}+\frac{20 f b_{1}}{21}+\frac{4 b_{1}^{2}}{7}\right) \\
& \times k^{4} P_{\operatorname{lin}}(k),
\end{aligned}
$$

where $c_{0}, c_{2}$, and $b_{4}$ are free fitting parameters and $b_{1}$ is the linear bias term which we explicitly inserted in Eq. (5.5) for future reference when we study redshift space galaxies. For dark matter $b_{1}=1$. Note that, unlike galaxies, the lowest order stochastic contribution to the dark matter redshift space power spectrum starts with the $k^{4}$ term [65], and hence, it is a higher order correction that can be ignored. The effects of these corrections are taken into account by the theoretical error.

The first goal of this section is to validate the inclusion of the next-to-leading order counterterm (5.4) into the oneloop theoretical models (5.1) and (5.2). Along the way, we will test the accuracy of the fingers-of-God modeling with phenomenological fitting functions that are often used in the literature; see e.g., [68]. These popular models approximate the fingers-of-God effect by a simple one-parameter Gaussian or Lorentzian damping, e.g.,

$$
P^{\mathrm{FOG}}(k, \mu)=\mathrm{e}^{-(k \mu \Sigma f)^{2}} P_{\mathrm{NL}}(k, \mu),
$$

where $\Sigma$ can be interpreted as a short-scale velocity dispersion. We stress that the phenomenological models such as (5.6) are not derived from first principles and introduce uncontrollable errors in parameter inference. To show this, we analyze the redshift space dark matter power spectrum data using the model (5.6) instead of the full effective field theory (EFT) description.

In the following analyses, for simplicity, we fix all cosmological parameters to their fiducial values except for $\sigma_{8}$, which is allowed to float freely. This choice is done only for simplicity. All our conclusions hold true even if we

\footnotetext{
${ }^{5} \mathrm{We}$ stress that this is a symbolic expression. Once IR resummation is included, it becomes impossible to write explicit close formulas for the Legendre integrals. In practice, we use the full expressions Eq. (5.2) and Eq. (5.4) and evaluate all Legendre integrals numerically with CLASS-PT [44].
}

vary all relevant cosmological parameters. We consider three models:

(a) the fitting function (5.6) applied to the one-loop SPT power spectrum, i.e., $P_{\mathrm{NL}}(k, \mu)=P_{\mathrm{SPT}}(k, \mu)$;

(b) the vanilla one-loop EFT with free $c_{0}, c_{2}$, but $b_{4}=0$;

(c) the one-loop EFT model with an additional NLO correction with free $b_{4}$.

Note that we implement the full IR resummation in case (a). In this setup the model (a) is a nonlinear resummation of perturbative models (b) and (c), which implies the following relationship between the counterterms:

$$
\begin{aligned}
& c_{0}=\frac{1}{210} f^{2} \Sigma^{2}\left(35 b_{1}^{2}+42 b_{1} f+15 f^{2}\right), \\
& c_{2}=\frac{1}{14} f \Sigma^{2}\left(7 b_{1}^{2}+12 b_{1} f+5 f^{2}\right), \\
& b_{4}=-\Sigma^{4} / 2 .
\end{aligned}
$$

Let us first discuss the $z=0$ snapshot. The resulting posterior distribution for nuisance parameters and clustering amplitude for the three models at $k_{\max }=0.1 \mathrm{hMpc}^{-1}$ are shown in Fig. 4 (see Appendix B for tables with 1D marginalized limits). The first relevant observation is that the approximate model (5.6) biases $\sigma_{8}$ by more than $5 \sigma$. However, varying $c_{0}$ and $c_{2}$ independently, one reduces the bias down to the $2 \sigma$ level. This illustrates that the phenomenological model with a single parameter $\Sigma$ fails to reproduce the data because it does not have enough freedom to describe the monopole and quadrupole simultaneously. Thus, keeping free coefficients in different counterterms is a crucial part of any reliable analysis. The residual bias in $\sigma_{8}$ is removed by adding the next-toleading order counterterm (5.4), which justifies its presence in the data analysis. This counterterm, however, does not allow us to increase the $k_{\max }$ range, which may be a signal of perturbation theory breakdown.

To demonstrate this, we perform the following exercise. We fix all cosmological parameters to their fiducial values and extract the best-fit parameters $c_{0}, c_{2}$, and $b_{4}$ from the data at $k_{\max }=0.1 \mathrm{hMpc}^{-1}$, where the fit is unbiased. Then we compare the contributions from leading and next-to-leading order counterterms of the quadrupole moment to the tree-level dark matter power spectrum evaluated for the best-fit parameters. We chose the quadrupole because this moment is most sensitive to the fingersof-God. The results are shown in the left panel of Fig. 5 . One can see that the NLO contribution surpasses the LO counterterm at $k \approx 0.13 \mathrm{hMpc}^{-1}$, and the whole tree-level expression at $k \approx 0.18 \mathrm{hMpc}^{-1}$. This can be naturally interpreted as a breakdown of the perturbative description for the dark matter in redshift space at $k \approx 0.15 h \mathrm{Mpc}^{-1}$ for $z=0$.

Now let us consider the $z=0.974$ snapshot. The relevant posterior distributions are shown in Fig. 6. One can observe the same trends as before: the fitting function (5.6) 


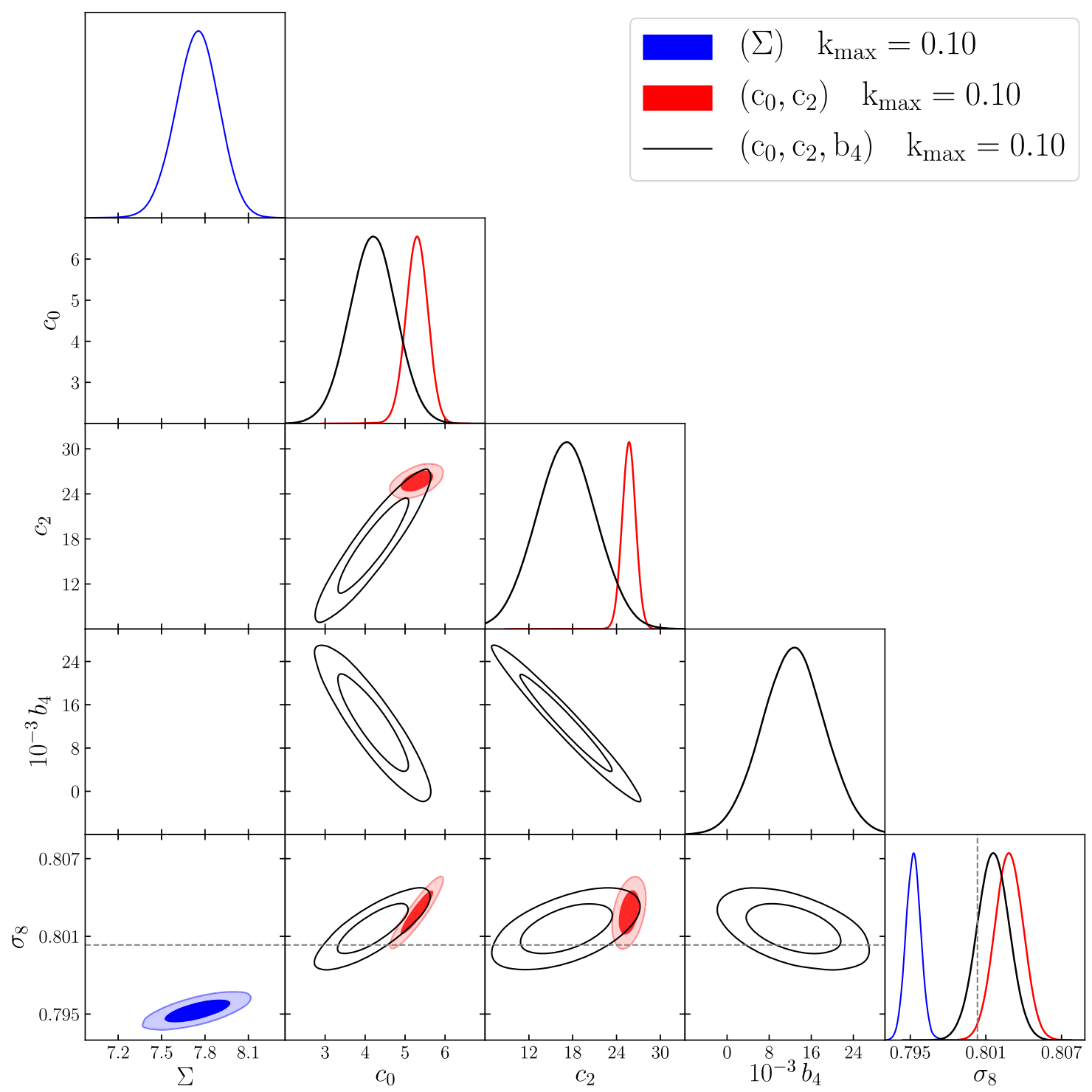

FIG. 4. Triangle plot for counterterm normalizations and $\sigma_{8}$ measured from the redshift space dark matter power spectrum of the Las Damas simulations at $z=0 . c_{0}, c_{2}, b_{4}$ are quoted in units $\left[h^{-1} \mathrm{Mpc}\right]^{2},\left[h^{-1} \mathrm{Mpc}\right]^{2},\left[h^{-1} \mathrm{Mpc}\right]^{4}$, respectively.
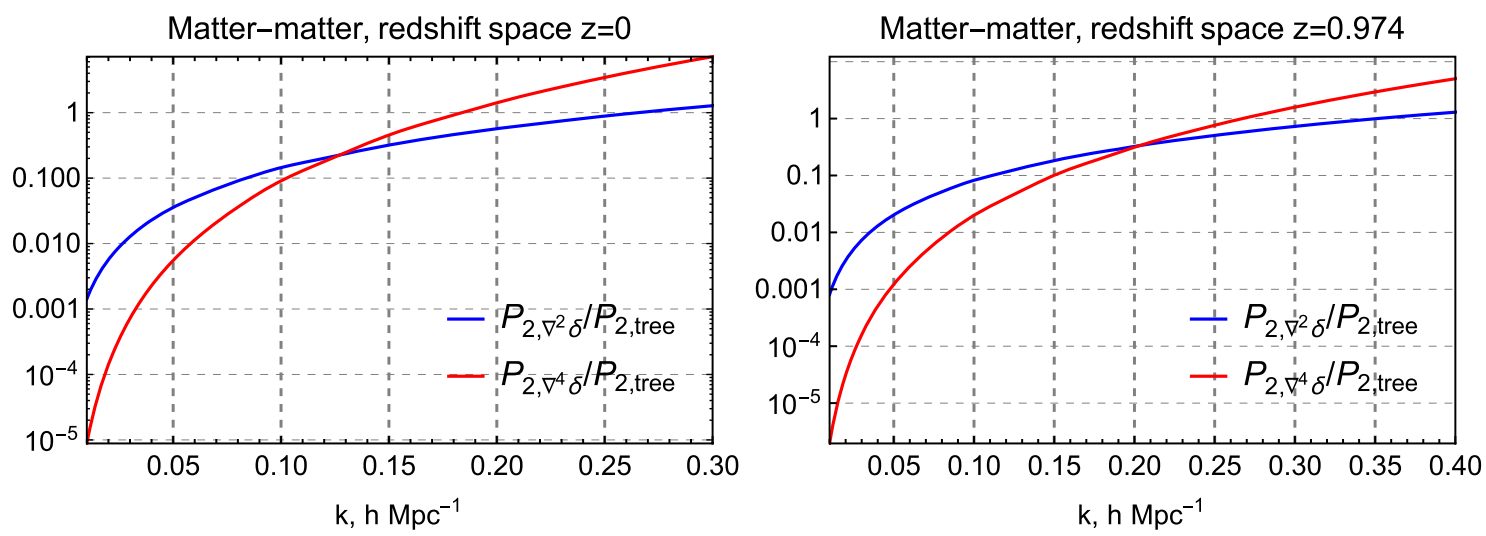

FIG. 5. The contribution of leading and next-to-leading order counterterm to quadrupole power spectrum for the best-fit parameters $c_{2}=17[\mathrm{Mpc} / h]^{2}, \quad b_{4}=1.3 \times 10^{4}[\mathrm{Mpc} / h]^{4}$ at $z=0$ (left panel) and $c_{2}=11[\mathrm{Mpc} / h]^{2}, \quad b_{4}=370[\mathrm{Mpc} / h]^{4}$ at $z=0.974$ (right panel). 


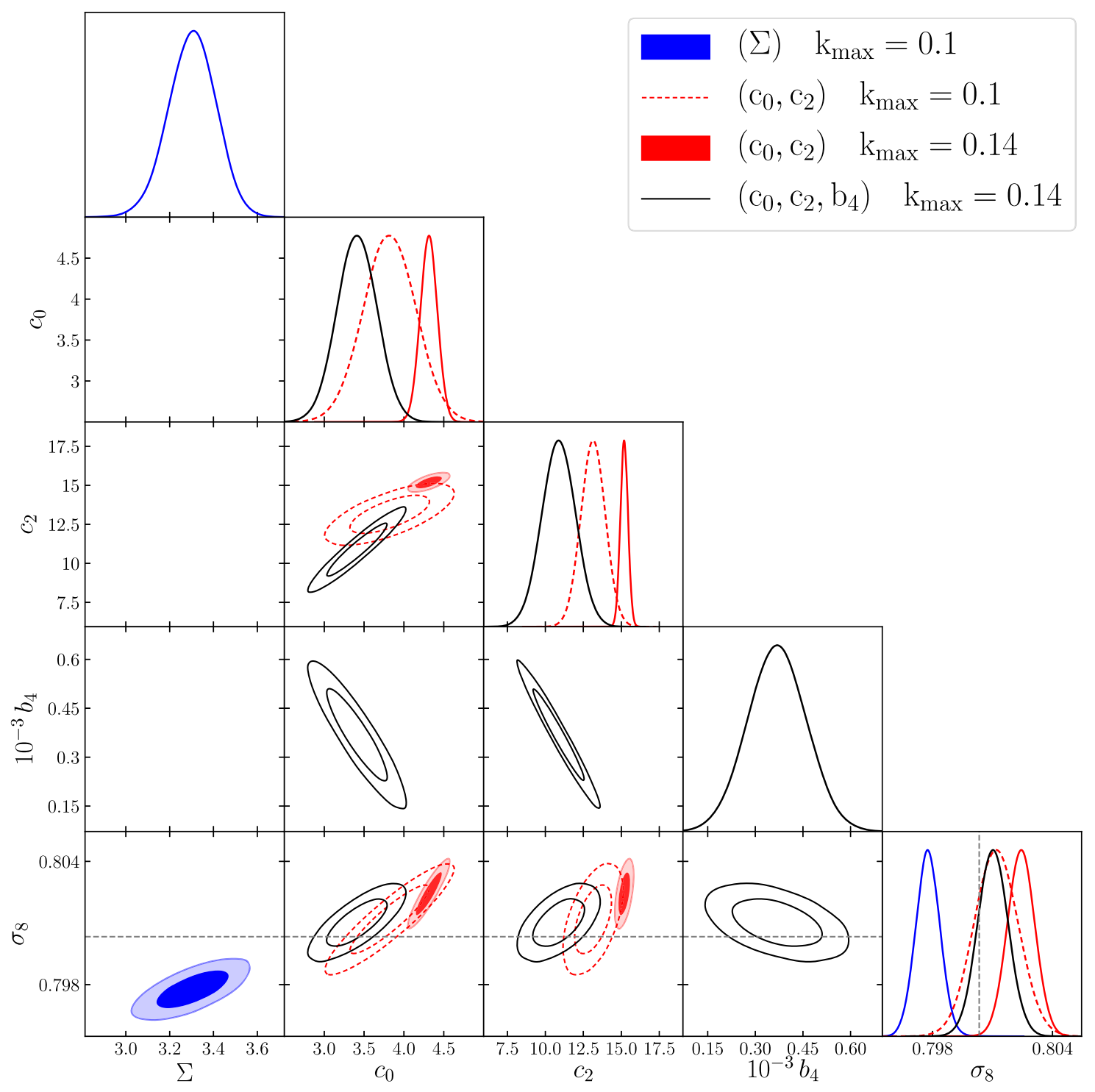

FIG. 6. Same as Fig. 4, but at redshift $z=0.974$.

[model (a)] yields a highly biased estimate of $\sigma_{8}$ already at $k_{\max }=0.1 \mathrm{~h} / \mathrm{Mpc}$. The naive EFT model (b) is accurate at $k_{\max }=0.1 \mathrm{~h} / \mathrm{Mpc}$, but fails to give a good fit beyond $k_{\max }=0.14 \mathrm{~h} / \mathrm{Mpc}$. The situation improves after the addition of the next-to-leading order counterterm $b_{4}$. We can clearly see that the presence of $b_{4}$ increases $k_{\max }$ and yields constraints better than the model (b) at $k_{\max }=0.1 \mathrm{hMpc}^{-1}$. This illustrates the crucial importance of fourth order short-scale velocity cumulant (5.4) for robust parameter inference. In what follows, we always include (5.4) in our baseline theoretical models (5.1) and (5.2). Finally, we confirm the validity of perturbation theory by plotting the LO and NLO quadrupole counterterms, normalized to the linear theory prediction, in the right panel of Fig. 5. We can see that the typical data cuts of our analysis $k_{\max }=(0.1-0.15) h \mathrm{Mpc}^{-1}$ are indeed lower than the nonlinear scale $k_{\mathrm{NL}, \mathrm{RSD}} \approx 0.25 \mathrm{hMpc}^{-1}$ at $z=0.974$.

One may wonder if a better model can be obtained by replacing $P_{\mathrm{NL}}$ in Eq. (5.6) computed in SPT by the EFT-like expression with one free counterterm,

$P^{\mathrm{FOG}}(k, \mu)=\mathrm{e}^{-(k \mu \Sigma f)^{2}}\left(P_{\mathrm{SPT}, 1-\text { loop }}(k, \mu)-2 c_{0}^{\prime} k^{2} P_{\text {lin }}(k)\right)$,

which can be referred to as the "EFT + FoG" model. In this case we have two free parameters and the resummed expression for the higher-derivative fingers-of-God corrections. We have found that both at $k_{\max }=0.1 \mathrm{hMpc}^{-1}$ and $k_{\max }=0.14 h \mathrm{Mpc}^{-1}$ this model is almost identical to the "vanilla EFT" case (b); see Fig. 18. This shows that the 


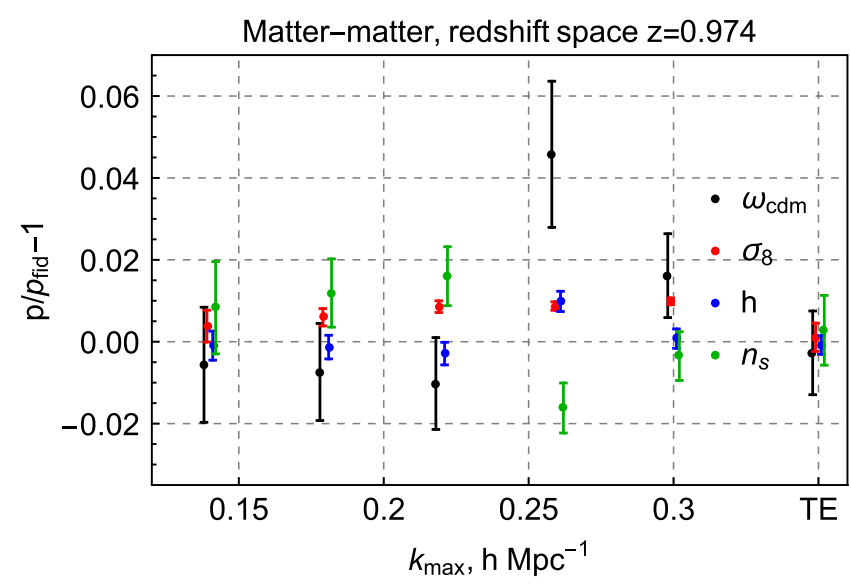

FIG. 7. Same as Fig. 1 for the redshift space matter power spectrum multipoles at $z=0.974$.

inclusion of the "FoG resummation" does not allow one to improve the fit over the basic EFT model. After including the $b_{4} k^{4} \mu^{4} P_{\text {lin }}$ term in the fit, we have found that the counterterm $b_{4}$ deviates from the prediction of the "resummation" formula $b_{4}^{\text {resum }}=-\Sigma^{4} / 2(5.7)$ at the $5 \sigma$ level. Note that even though $b_{4}^{\text {resum }}$ has the same order of magnitude as the actual $b_{4}$ measured from the data, its sign is wrong. This shows that the perturbative EFT expansion with the $k^{4} \mu^{4} P_{\text {lin }}$ counterterm provides a better model than the resummed nonlinear damping function given in Eq. (5.6). This conclusion should also hold true for any nonlinear damping function that predicts a positive coefficient in front of the $k^{4} \mu^{4} P_{\text {lin }}$ term, e.g., the Lorentzian damping.

Let us summarize the results of this section so far. First, we have shown that the use of overconstrained fitting formulas for the fingers-of-God can generate significant bias in the inferred cosmological parameters. Second, we have justified the inclusion of the next-to-leading order $k^{4} \mu^{4} P_{\text {lin }}$ counterterm, by showing that it allows us to extend the regime of applicability of the EFT description and improve the parameter constraints.

\section{B. Cosmological parameters}

We present now the complete cosmological analysis of the redshift space dark matter power spectrum in the $k_{\max }$ and TE analyses.

In the previous section we have seen that the perturbative description breaks down at $z=0$ already on quite large scales $k \sim 0.1 \mathrm{hMpc}^{-1}$, and hence this case may not be very illustrative. We focus on the $z=0.974$ snapshot in what follows.

The marginalized 1D constraints on cosmological parameters for different momentum cuts and the theoretical error are shown in Fig. 7. The theory prediction for TE
TABLE II. The marginalized 1D intervals for the cosmological and nuisance parameters estimated from the monopole and quadrupole moments of the Las Damas dark matter redshift space power spectrum at $z=0.974$. We show the fitted parameters (first column), fiducial values used in simulations (second column), the resulting parameter constraints for the baseline $k_{\max }$ analysis (third column), and the theoretical error approach (fourth column). $c_{0}, c_{2}, b_{4}$ are quoted in units $\left[h^{-1} \mathrm{Mpc}\right]^{2},\left[h^{-1} \mathrm{Mpc}\right]^{2}$, $\left[h^{-1} \mathrm{Mpc}\right]^{4}$, respectively.

\begin{tabular}{lccc}
\hline \hline & & \multicolumn{2}{c}{$z=0.974$} \\
\cline { 3 - 4 } Par & Fid & $k_{\max }=0.14 h / \mathrm{Mpc}$ & $\mathrm{TE}$ \\
\hline$\omega_{\mathrm{cdm}}$ & 0.1029 & $0.1023_{-1.5 \times 10^{-3}}^{+1.4 \times 10^{-3}}$ & $0.1026_{-1.0 \times 10^{-3}}^{+1.1 \times 10^{-3}}$ \\
$h$ & 0.7 & $0.6993_{-2.6 \times 10^{-3}}^{+2.4 \times 10^{-3}}$ & $0.6995_{-1.6 \times 10^{-3}}^{+1.6 \times 10^{-3}}$ \\
$n_{s}$ & 1 & $1.0083_{-0.011}^{+0.012}$ & $1.0028_{-8.5 \times 10^{-3}}^{+8.5 \times 10^{-3}}$ \\
$A$ & 1 & $1.0096_{-0.016}^{+0.016}$ & $1.0043_{-0.011}^{+0.010}$ \\
$\Omega_{m}$ & 0.25 & $0.2493_{-1.8 \times 10^{-3}}^{+1.7 \times 10^{-3}}$ & $0.2498_{-1.3 \times 10^{-3}}^{+1.3 \times 10^{-3}}$ \\
$\sigma_{8}$ & 0.8003 & $0.8033_{-3.1 \times 10^{-3}}^{+3.1 \times 1.10}$ & $0.8012_{-2.8 \times 10^{-3}}^{+2.8 \times 10^{-3}}$ \\
$c_{0}$ & $\ldots$ & $3.66_{-0.37}^{+0.39}$ & $3.29_{-0.39}^{+0.40}$ \\
$c_{2}$ & $\ldots$ & $11.26_{-1.25}^{+1.32}$ & $10.52_{-1.10}^{+1.11}$ \\
$10^{-3} b_{4}$ & $\cdots$ & $0.34_{-0.11}^{+0.10}$ & $0.41_{-0.08}^{+0.08}$ \\
\hline \hline
\end{tabular}

analysis was computed at fiducial $k_{\max }^{\mathrm{fid}}=0.12 \mathrm{~h} / \mathrm{Mpc}$. We found that the momentum cutoff $k_{\max }=0.14 h / \mathrm{Mpc}$ reproduces the true parameters within the $1 \sigma$ interval. However, the clustering amplitude becomes biased by more than $2 \sigma$ starting from $k_{\max }=0.16 \mathrm{~h} / \mathrm{Mpc}$. Given this reason, we choose $k_{\max }=0.14 \mathrm{~h} / \mathrm{Mpc}$ as our baseline data cut. The final constraints on cosmological and nuisance parameters for this $k_{\max }$ and for the theoretical error analysis are displayed in Table II and in Fig. 8. As in the previous section, we note three key features of the theoretical error covariance. First, we can obtain the cosmological constraints with a single MCMC analysis. This can be contrasted with the standard approach that requires running many analyses with different choices of $k_{\max }$. Second, we get unbiased estimates for cosmological parameters and reliable error bars. Third, these error bars happened to be smaller than those obtained in the $k_{\max }$ analyses; the constraints on $\omega_{\mathrm{cdm}}, h, n_{s}$, and $\sigma_{8}$ improve by $30 \%, 40 \%, 25 \%, 10 \%$, respectively. This happens mainly due to additional $\mathrm{BAO}$ wiggles, which are included beyond $k_{\max }=0.14 \mathrm{hMpc}^{-1}$. Indeed, we can see that the 2D probability distribution in the $\omega_{\mathrm{cdm}}-h$ plane, which reflects the BAO signal, is significantly narrower in the TE analysis.

Given some significant improvements that we have obtained, it is important to check whether they remain if we alter the theoretical error envelope. The details of this analysis are presented in Appendix C. We have found that the parameter constraints do depend on the choice of fiducial cosmology quite noticeably. This implies that in 


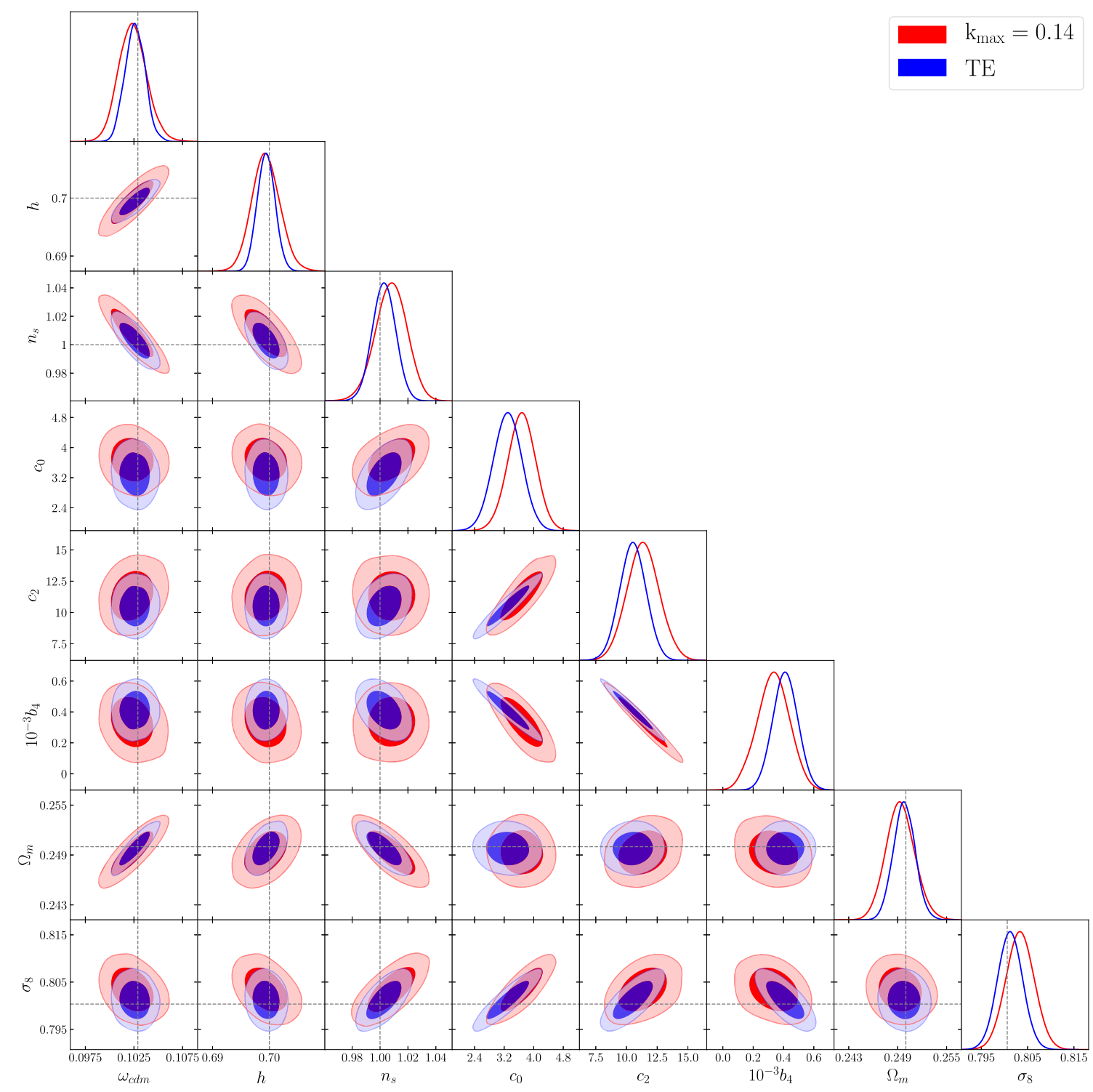

FIG. 8. Triangle plot for the cosmological and nuisance parameters measured from the redshift space dark matter power spectrum of the Las Damas simulations at $z=0.974 . c_{0}, c_{2}, b_{4}$ are quoted in units $\left[h^{-1} \mathrm{Mpc}\right]^{2},\left[h^{-1} \mathrm{Mpc}\right]^{2},\left[h^{-1} \mathrm{Mpc}\right]^{4}$, respectively.

the regime where the constraints are dominated by the theoretical error one has to be careful about its accurate modeling.

\section{GALAXIES IN REAL SPACE}

In this section we focus on the galaxy clustering in real space. Although this analysis is academic in nature, it will allow us to assess the validity of the perturbative bias model and clearly see the implications of the theoretical error for biased tracers. We will use the effective field theory model characterized by the following set of nuisance parameters (see [44] for our notations):

$$
\left\{b_{1}, b_{2}, b_{\mathcal{G}_{2}}, R_{*}^{2}, b_{\Gamma_{3}}, P_{\text {shot }}\right\}
$$

which includes the local quadratic bias $b_{2}$, the quadratic tidal bias $b_{\mathcal{G}_{2}}$, the higher-derivative counterterm ${ }^{6} R_{*}^{2}$, and the constant shot noise contribution $P_{\text {shot }}$. Even though the paircounting shot noise prediction $\bar{n}^{-1}$ was subtracted from the power spectrum data, we still need to keep a constant nuisance parameter in the fit because the value of shot noise is, in general, expected to deviate from $\bar{n}^{-1}$ due to exclusion effects [69] (or fiber collisions in realistic surveys [70]). These deviations can be as large as $\sim 30 \%$ of $\bar{n}^{-1}$ for the BOSS-like host halos [71]. Given this reason, we marginalize over $P_{\text {shot }}$ within the following Gaussian prior:

\footnotetext{
${ }^{6}$ Since the degeneracy between $R_{*}^{2}$ and $c_{s}^{2}$ (in the notation of [44]) cannot be broken at the power spectrum level, we fix the dark matter counterterm $c_{s}^{2}=1\left[h^{-1} \mathrm{Mpc}\right]^{2}$ to avoid clutter.
} 


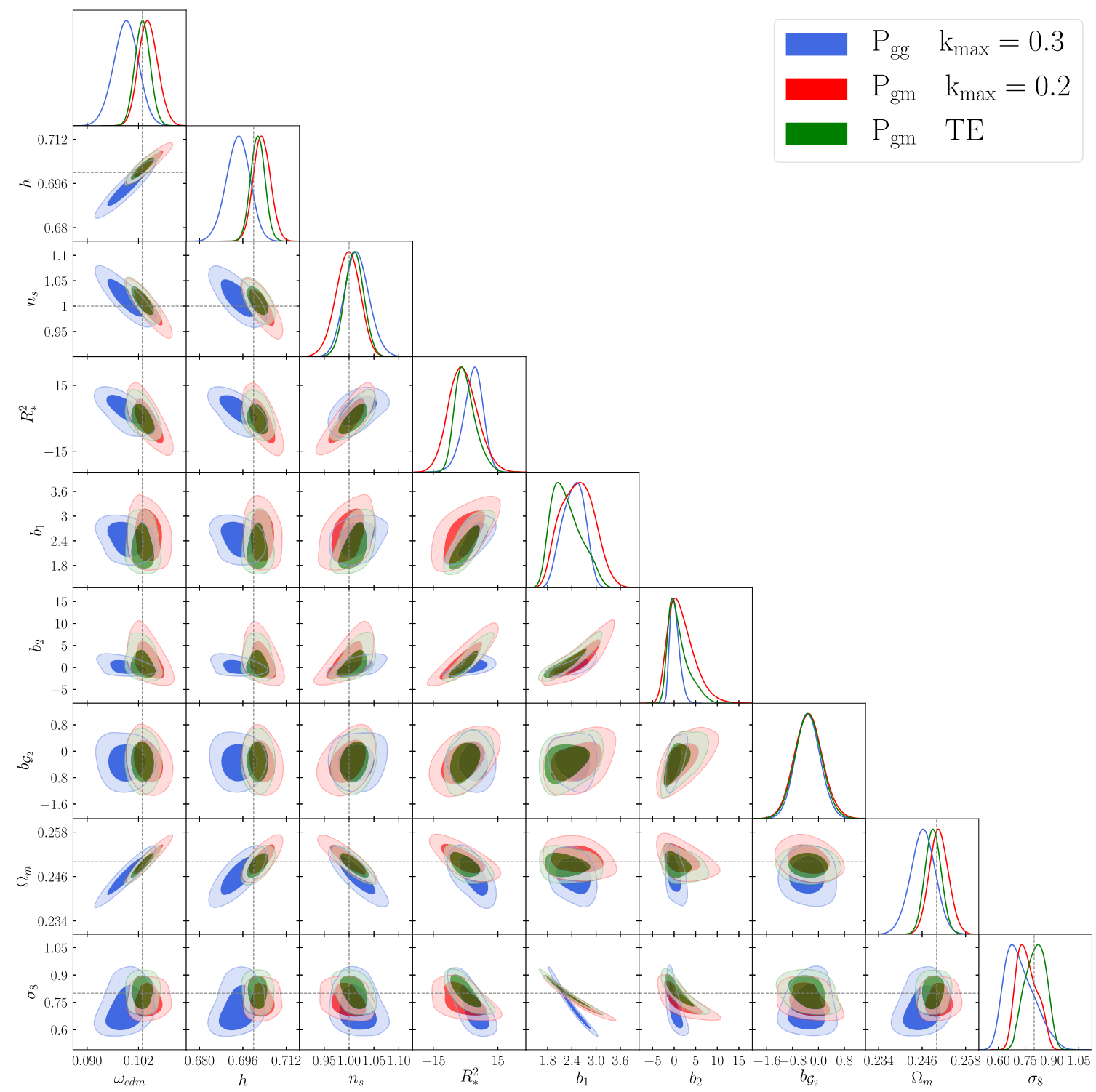

FIG. 9. Triangle plot for the cosmological and nuisance parameters measured from the real space galaxy power spectrum of the Las Damas simulations at $z=0.342$.

$$
P_{\text {shot }} \sim\left(0,\left(0.3 \cdot \bar{n}^{-1}\right)^{2}\right) .
$$

Note that the value of the residual shot noise contribution can be negative. We do not impose any priors on the other biases and counterterms, except for the cubic tidal bias $b_{\Gamma_{3}}$, which we found to be very degenerate with $b_{\mathcal{G}_{2}}$. We marginalize over $b_{\Gamma_{3}}$ assuming the physical prior centered at the prediction of the coevolution model [72,73],

$$
b_{\Gamma_{3}}^{(\text {Coev })}=\frac{23}{42}\left(b_{1}-1\right)=0.66 \text { for } b_{1}=2.2,
$$

and with the unit Gaussian variance,

$$
b_{\Gamma_{3}} \sim \mathcal{N}\left(b_{\Gamma_{3}}^{(\mathrm{Coev})}, 1^{2}\right) .
$$

Finally, we have checked that the data do not show any evidence for the scale-dependent stochastic contributions $a_{0} k^{2}$ for $k \lesssim 0.3 \mathrm{hMpc}^{-1}$, and hence we did not use it in our model. This is consistent with the results of $N$-body simulations done in Ref. [71].

We analyzed the galaxy-galaxy power spectrum and the galaxy-matter cross-spectrum at $z=0.342$ using the $k_{\max }$ approach and found that the posterior distributions are unbiased for all $k_{\max }$ up to $k_{\mathrm{NL}} \simeq 0.3 \mathrm{hMpc}^{-1}$. We do not push to higher $k$ 's because the perturbative expansion is clearly not valid there. The resulting posterior distributions for nuisance and cosmological parameters are shown in Fig. 9 (blue contours), and the 1D marginalized parameter limits are presented in Table III. 
TABLE III. The marginalized 1D intervals for the cosmological parameters estimated from the Las Damas real space galaxy power spectrum and galaxy-matter cross-spectrum at $z=0.342$. Shown are the fitted parameters (first column), the fiducial values used in simulations (second column), the results for $P_{\mathrm{gg}}$ at $k_{\max }=0.3 \mathrm{hMpc} \mathrm{Mp}^{-1}$ (third column), the results for $P_{\mathrm{gm}}$ at $k_{\max }=0.2 \mathrm{hMpc}^{-1}$ (fourth column), and the results for $P_{\mathrm{gm}}$ with theoretical error approach (fifth column).

\begin{tabular}{lcccc}
\hline \hline Par & Fid & $\mathrm{P}_{\mathrm{gg}} k_{\max }=0.3 \mathrm{~h} / \mathrm{Mpc}$ & $\mathrm{P}_{\mathrm{gm}} k_{\max }=0.2 \mathrm{~h} / \mathrm{Mpc}$ & $\mathrm{P}$ gm $\mathrm{TE}$ \\
\hline$\omega_{\mathrm{cdm}}$ & 0.1029 & $0.099_{-2.9 \times 10^{-3}}^{+3.0 \times 10^{-3}}$ & $0.1043_{-2.4 \times 10^{-3}}^{+2.1 \times 10^{-3}}$ & $0.1030_{-1.9 \times 10^{-3}}^{+1.8 \times 10^{-3}}$ \\
$h$ & 0.7 & $0.6942_{-4.3 \times 10^{-3}}^{+4.6 \times 10^{-3}}$ & $0.7031_{-3.3 \times 10^{-3}}^{+3.1 \times 10^{-3}}$ & $0.7015_{-2.7 \times 10^{-3}}^{+2.7 \times 10^{-3}}$ \\
$n_{s}$ & 1 & $1.0160_{-0.026}^{+0.024}$ & $0.9973_{-0.024}^{+0.026}$ & $1.0104_{-0.019}^{+0.019}$ \\
$A$ & 1 & $0.8570_{-0.2509}^{+0.1085}$ & $0.8919_{-0.184}^{+0.092}$ & $1.0157_{-0.157}^{+0.157}$ \\
$\Omega_{m}$ & 0.25 & $0.2462_{-3.3 \times 10^{-3}}^{+3.6 \times 10^{-3}}$ & $0.2506_{-2.8 \times 10^{-3}}^{+2.6 \times 10^{-3}}$ & $0.2490_{-2.3 \times 10^{-3}}^{+2.2 \times 10^{-3}}$ \\
$\sigma_{8}$ & 0.8003 & $0.7208_{-0.108}^{+0.057}$ & $0.7600_{-0.076}^{+0.043}$ & $0.8080_{-0.052}^{+0.072}$ \\
$R_{*}^{2}$ & $\ldots$ & $3.46_{-3.93}^{+4.97}$ & $-1.09_{-7.21}^{+6.02}$ & $-0.03_{-5.54}^{+3.60}$ \\
$b_{1}$ & $\ldots$ & $2.44_{-0.26}^{+0.32}$ & $2.54_{-0.51}^{+0.41}$ & $2.26_{-0.48}^{+0.24}$ \\
$b_{2}$ & $\ldots$ & $0.17_{-1.34}^{+0.81}$ & $1.74_{-3.79}^{+2.03}$ & $1.04_{-2.27}^{+1.92}$ \\
$b_{\mathcal{G}_{2}}$ & $\ldots$ & $-0.326_{-0.397}^{+0.376}$ & $-0.309_{-0.457}^{+0.425}$ & $-0.315_{-0.423}^{+0.415}$ \\
\hline \hline
\end{tabular}

All in all, we do not see any evidence for the theoretical error up to very high $k_{\max }$ for the power spectrum of galaxies in real space. We believe that it is caused by the following two reasons. First, the statistical covariance for galaxies contains a large shot noise contribution, which slows down the reduction of the power spectrum errors on short scales compared to the dark matter case studied before. Thus, unlike the dark matter case, the theoretical error for galaxies is always smaller than the statistical one on the scales of interest. Note that the situation can be different for another type of galaxies, whose shot noise is lower, e.g., the DESI bright galaxy sample [10]. The second reason is a large number of fitting parameters: the real space galaxy power spectrum data alone cannot efficiently break the degeneracies between these parameters. An example is the strong degeneracy $b_{1}-\sigma_{8}$, which also generates a highly not-Gaussian posterior distribution of $\sigma_{8}$ seen in Fig. 9 .

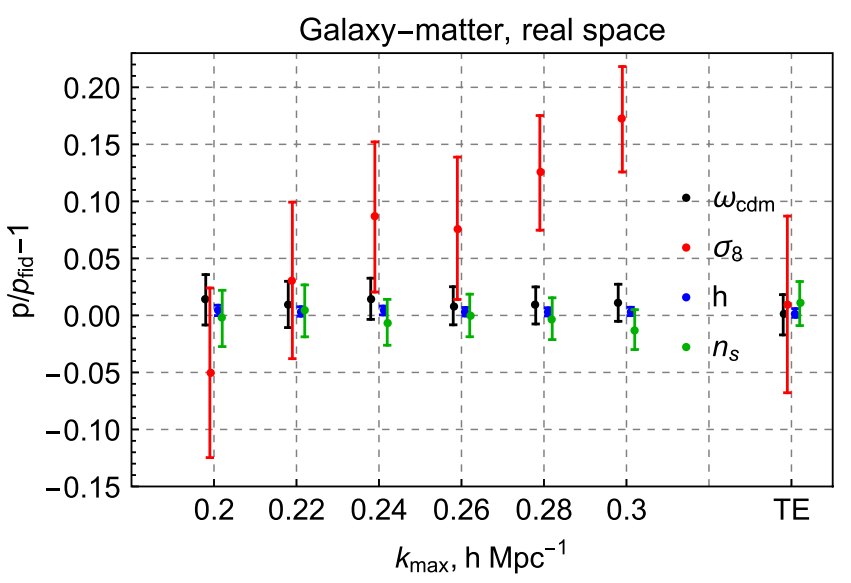

FIG. 10. Marginalized 1D limits on cosmological parameters inferred from the real-space galaxy-matter cross-spectrum as a function of $k_{\max }$. The rightmost points result from the TE analysis. All parameters are normalized to their fiducial values.
Indeed, these two effects can be clearly assessed by analyzing the galaxy-matter cross-spectrum $P_{\mathrm{gm}}$. The shot noise contribution to the covariance matrix is reduced for $P_{\text {gm }}$ [see Eq. (3.3)], and there is no constant shot noise contribution $P_{\text {shot }}$ in the fit, which narrows the posterior distribution compared to the $P_{\mathrm{gg}}$ case for the same $k_{\max }$. The 1D marginalized limits on the cosmological parameters as a function of $k_{\max }$ are shown in Fig. 10. The theory prediction for TE analysis was computed at fiducial $k_{\max }^{\mathrm{fid}}=0.18 h / \mathrm{Mpc}$.

We see that the 1D posterior distributions for $P_{\mathrm{gm}}$ are unbiased up to $k_{\max } \approx 0.22 \mathrm{hMpc}^{-1}$. However, a closer inspection of the MCMC results revealed a bias in the 2D posterior contours. This suggests that using the marginalized 1D distributions to select $k_{\max }$ can be misleading: one has to ensure that not only the 1D marginalized constraints are unbiased, but also the 2D contours enclose the fiducial cosmology, e.g., within $68 \%$ C.L. Indeed, for some data cuts the principal components of certain parameters can be biased, but their projections onto particular parameter planes can accidentally fall close to the fiducial values. Using such data cuts can lead to biases when different probes are combined, as in this case the resulting posterior distributions are driven by the degeneracy breaking between different principal components (PCs) of the combined datasets. This motivated us to choose $k_{\max }=$ $0.2 \mathrm{hMpc}^{-1}$ as a baseline data cut for $P_{\mathrm{gm}}$.

Since the baseline $k_{\max }$ is quite low, we expect to gain some information with the theoretical error. The posterior distributions for the $k_{\max }$ and the TE cases are shown in Fig. 9, while the parameter limits are presented in Table III. Just as in the dark matter case, the TE covariance sharpens the constraints on the parameters related to the power spectrum shape and the BAO: $\omega_{\mathrm{cdm}}, h$, and $n_{s}$ improve by $20 \%, 20 \%$, and $25 \%$, respectively. In contrast, the 
constraint on $\sigma_{8}$ is not significantly affected. Note also that the mean $\sigma_{8}$ shifts toward the true fiducial value. The constraint on $\sigma_{8}$ does not improve due to the notorious degeneracy $b_{1}-\sigma_{8}$. Indeed, the linear-theory degeneracy $b_{1}-\sigma_{8}$ can be broken only by the one-loop corrections. This makes the result very sensitive to the largest wave number bins used in the analysis, because for these bins the amplitude of the loop corrections is large. However, when the one-loop correction becomes large, the two-loop contribution becomes non-negligible as well. The theoretical error covariance is exactly introduced to alleviate this problem and marginalize over all possible two-loop shapes. It does not sharpen the constraints on $\sigma_{8}$, but yields some improvement for other parameters related to the shape and the BAO.

One possible way to shrink the posterior distribution in the galaxy power- and cross-spectra cases is to fix some bias parameters to the predictions of some phenomenological models, i.e., the coevolution model for the dark matter halos [72]. However, this can result in overfitting and wrong estimation of the parameter error bars. We believe that a more appropriate approach is to vary all relevant nuisance parameters in the fit within physical priors, as we do in this paper. In a realistic survey one always observes galaxies in redshift space, which allows for breaking of the $b_{1}-\sigma_{8}$ degeneracy through the redshift space distortions. Let us now consider the latter case.

\section{GALAXIES IN REDSHIFT SPACE}

In this section we scrutinize the galaxy clustering in redshift space in the context of the theoretical error covariance. We will analyze the monopole and quadrupole moments of the redshift space galaxy power spectrum at $z=0.342$. The redshift of this sample is somewhat lower than the effective redshifts of future surveys; hence this represents the stringent test of our approach.

The EFT model with NLO fingers-of-God corrections is characterized by the following set of nuisance parameters (see Ref. [11] for our conventions):

$$
\left\{b_{1}, b_{2}, b_{\mathcal{G}_{2}}, b_{\Gamma_{3}}, P_{\text {shot }}, c_{0}, c_{2}, b_{4}\right\} .
$$

We use the same priors on $b_{\Gamma_{3}}$ and $P_{\text {shot }}$ as in the previous section, as well as infinitely large flat priors for other parameters. Note that on general grounds one can expect the presence of the additional stochastic contribution $P_{\text {stoch }}(k, \mu)=a_{2} \mu^{2} k^{2}$. However, we have found that this contribution is completely degenerate with $b_{4}$ for the $P_{0}+P_{2}$ data vector, which motivated us to fix $a_{2}=0$ as in Refs. [31,44].

As a first step, we run a sequence of analyses varying the data cut $k_{\max }$. The 1D marginalized constraints on the cosmological parameters as functions of $k_{\max }$ are presented in Fig. 11. The rightmost points correspond to the theoretical error analysis with the cutoff $k_{\max }=0.32 \mathrm{~h} / \mathrm{Mpc}$. One may observe that the standard analyses yields a biased estimate for

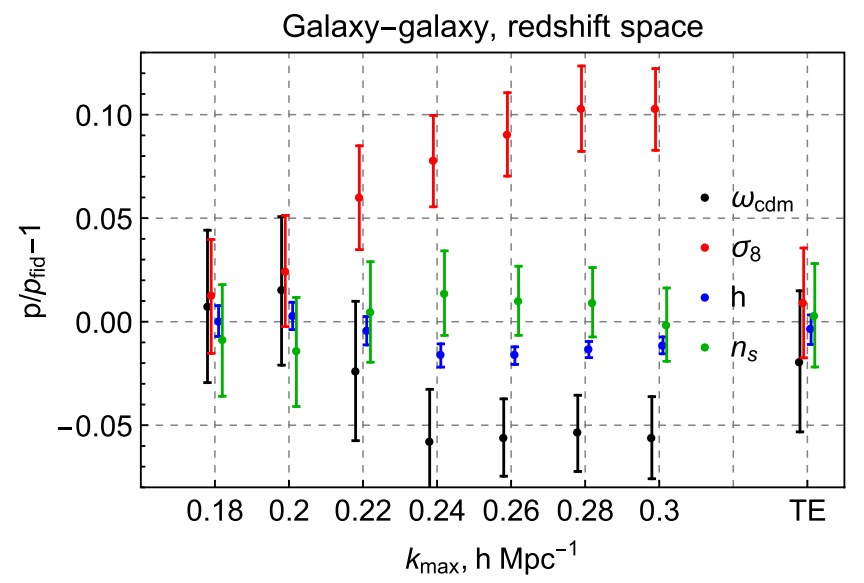

FIG. 11. Same as Fig. 10, but for the redshift space galaxy multipoles.

$\sigma_{8}$ for $k_{\max }>0.20 h / \mathrm{Mpc}$. Shortly after, $\omega_{\text {cdm }}$ and $h$ start deviating from the true values at $k_{\max } \approx 0.23 \mathrm{~h} / \mathrm{Mpc}$.

This motivates us to choose $k_{\max }=0.18 \mathrm{~h} / \mathrm{Mpc}$ as our baseline analysis with the sharp momentum cutoff as in this case all marginalized posteriors contain the true values well within $1 \sigma$.

The values of the marginalized 1D constraints for the baseline $k_{\max }$ and the TE analyses are shown in Table IV. The 2D posteriors are presented in Fig. 12. The theory prediction for TE analysis was computed at fiducial $k_{\max }^{\mathrm{fid}}=0.16 h / \mathrm{Mpc}$.

Overall, we see that for the realistic example of redshift space galaxies the theoretical error approach improves the parameter constraints over the $k_{\max }$ case quite modestly

TABLE IV. The marginalized 1D intervals for the cosmological parameters estimated from the Las Damas redshift space galaxy power spectra at $z=0.342$. The table contains fitted parameters (first column), the fiducial values used in simulations (second column), the results of the baseline $k_{\max }$ analysis (third column), and the outcome of the theoretical error approach (fourth column). $\quad c_{0}, \quad c_{2}, \quad b_{4}$ are quoted in units $\left[h^{-1} \mathrm{Mpc}\right]^{2}$, $\left[h^{-1} \mathrm{Mpc}\right]^{2},\left[h^{-1} \mathrm{Mpc}\right]^{4}$, respectively.

\begin{tabular}{lccc}
\hline \hline Par & Fid & $k_{\max }=0.18 h / \mathrm{Mpc}$ & TE \\
\hline$\omega_{\mathrm{cdm}}$ & 0.1029 & $0.1037_{-4.1 \times 10^{-3}}^{+3.5 \times 10^{-3}}$ & $0.1009_{-3.5 \times 10^{-3}}^{+3.5 \times 10^{-3}}$ \\
$h$ & 0.7 & $0.7002_{-5.3 \times 10^{-3}}^{+5.1 \times 3}$ & $0.6973_{-5.0 \times 10^{-3}}^{+5.0 \times 10^{-3}}$ \\
$n_{s}$ & 1 & $0.9909_{-0.026}^{+0.028}$ & $1.0031_{-0.025}^{+0.025}$ \\
$A$ & 1 & $1.0169_{-0.079}^{+0.073}$ & $1.0571_{-0.081}^{+0.069}$ \\
$\Omega_{m}$ & 0.25 & $0.2513_{-5.2 \times 10^{-3}}^{+4.3}$ & $0.2479_{-4.7 \times 10^{-3}}^{+4.3 \times 10^{-3}}$ \\
$\sigma_{8}$ & 0.8003 & $0.8069_{-0.022}^{+0.022}$ & $0.8044_{-0.021}^{+0.021}$ \\
$c_{0}$ & $\ldots$ & $0.77_{-11.48}^{+17.93}$ & $9.40_{-9.68}^{+12.45}$ \\
$c_{2}$ & $\ldots$ & $36.22_{-20.61}^{+28.01}$ & $29.61_{-18.30}^{+23.11}$ \\
$10^{-3} b_{4}$ & $\ldots$ & $1.40_{-0.26}^{+0.21}$ & $1.73_{-0.28}^{+0.25}$ \\
$b_{1}$ & $\ldots$ & $2.17_{-0.07}^{+0.07}$ & $2.16_{-0.07}^{+0.07}$ \\
$b_{2}$ & $\ldots$ & $-0.96_{-0.87}^{+0.64}$ & $-0.92_{-0.78}^{+0.57}$ \\
$b_{\mathcal{G}_{2}}$ & $\ldots$ & $-0.350_{-0.388}^{+0.364}$ & $-0.330_{-0.368}^{+0.351}$ \\
\hline \hline
\end{tabular}




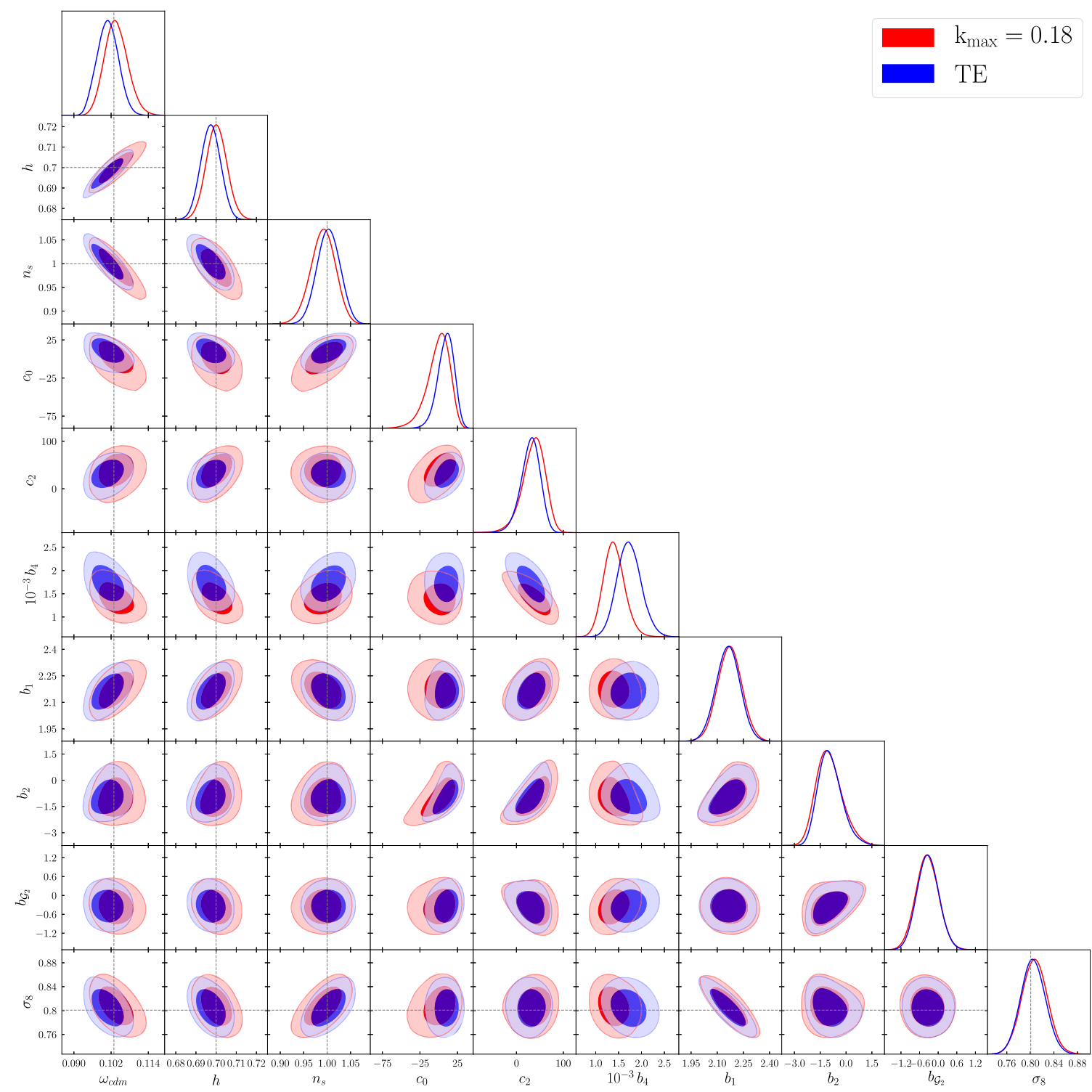

FIG. 12. Triangle plot for the cosmological and nuisance parameters measured from the redshift space galaxy power spectrum of the Las Damas simulations at $z=0.342 . c_{0}, c_{2}, b_{4}$ are quoted in units $\left[h^{-1} \mathrm{Mpc}\right]^{2},\left[h^{-1} \mathrm{Mpc}\right]^{2},\left[h^{-1} \mathrm{Mpc}\right]^{4}$, respectively.

$(\lesssim 10 \%)$, unlike the previous case of the galaxy-matter cross-spectrum. This happens because the $\mathrm{BAO}$ wiggles at $k>0.18 \mathrm{hMpc}^{-1}$, neglected in the $k_{\max }$ analysis, are strongly suppressed due to redshift space displacements [40] and the large shot noise covariance [cf. Eq. (3.3) and Eq. (3.4)]. There is, however, some non-negligible improvement for the nuisance parameters, whose values are quite sensitive to the large- $k$ modes. As far as the cosmological parameters are concerned, qualitatively, we can conclude that the TE covariance, if properly chosen, automatically optimizes the choice of $k_{\max }$ and guarantees that parameter limits are unbiased, but does not noticeably improve them. This implies that the application of the theoretical error approach most likely will not sharpen the cosmological constraints from the current surveys like BOSS, where $k_{\max }$ has already been measured for various analysis settings $[1,30]$.
Comparing the cases of redshift space dark matter and redshift space galaxies, we can understand the reason why the gain from the theoretical error is so modest in the latter case. The main difference between these two cases is the presence of four additional shapes in the nonlinear bias model, which also act like theoretical error; see Sec. II. Hence, the addition of an extra theoretical error shape does not have a large impact on the likelihood because it already contains the theoretical error due to marginalization over the nonlinear bias parameters.

Finally, it is important to point out that we have checked that our results for galaxies in redshift space are invariant with respect to the choice of the theoretical error template; see Appendix C. This is an important consistency check which validates the theoretical error approach for realistic analyses. 


\section{CONCLUSIONS}

We have validated the theoretical error approach on large-volume $N$-body simulations data for the clustering of dark matter and galaxies in real and redshift space. First, we introduced a new mock-based approach to extract the theoretical error covariance from the data. This approach allowed us to avoid uncertainties in the theoretical estimates of higher-order nonlinearities. We have argued that the calibration of the theoretical error from the $N$-body mock simulations can be the optimal strategy to build the theoretical error likelihood.

We have demonstrated that the use of the TE covariance in the power spectrum likelihoods allows us to recover the input cosmological parameters used in the simulations of dark matter and galaxies in real and redshift spaces. Crucially, using the TE approach we can avoid lengthy measurements of $k_{\max }$ and perform the full parameter inference with a single MCMC run. To put this result in context, let us consider the case of the recent analysis of the BOSS data from Ref. [30]. This work calibrated their baseline $k_{\max }$ by analyzing $N$-body simulation data for four different choices of the data cut. Achieving good convergence for nuisance and cosmological parameters for these chains requires $184000 \mathrm{CPU}$ hours on our cluster, which can be saved if the theoretical error approach is implemented. We stress that all this CPU time is required for only one particular choice of priors and fitted parameters. If a different set of priors or model parameters are used, the data cut $k_{\max }$ needs to be remeasured, as explained in detail in Ref. [30]. Hence, the actual computational saving from the theoretical error likelihood can be even larger.

We have also performed a detailed comparison of the TE results with the $k_{\max }$ analyses. For dark matter in real and redshift space, cosmological constraints actually improve with the theoretical error covariance. This happens because it allows us to extract the information encoded in the BAO wiggles at wave numbers larger than $k_{\max }$, while marginalizing over the broadband uncertainties.

For galaxies, the presence of the large shot noise term in the covariance and proliferation of nuisance parameters inflate the error bars and force us to use more aggressive data cuts, where the BAO information is saturated. Due to this reason, the TE approach does not significantly improve the parameter limits unlike in the dark matter case. For the galaxy autospectrum in real space, we have not found any evidence for theoretical error up to the nonlinear scale, and hence the TE approach is fully identical to the standard $k_{\max }$ analysis. Finally, for galaxies in redshift space, the theoretical error is nonvanishing and its inclusion in the covariance matrix effectively optimizes the choice of $k_{\max }$ at which the inferred parameters are unbiased. The cosmological parameter constraints improve quite marginally in this case, unlike the nuisance parameters, whose posterior volume shrinks appreciably.

As a by-product, we have validated the effective field theory implementation of the CLASS-PT code [44] by showing that it can accurately reproduce the true fiducial parameters from various power spectrum data vectors used in this paper. In passing, we have shown that the use of overconstrained nonlinear fingers-of-God models leads to biases in parameter inferences. Besides, we have advocated the inclusion of the higher-order counterterm in the description of nonlinear redshift space distortions. Indeed, it allows us to extend the range of scales where the modeling is accurate and eventually to improve the cosmological constraints as compared to the standard one-loop effective field theory model without the higher-order corrections. This justifies the analyses of Refs. [1,5,31] that included the higher derivative fingersof-God correction.

It is worth pointing out that in this paper we have studied the mock galaxies that simulate the BOSS LRG sample [55]. This sample exhibits large fingers-of-God features, which do not allow us to extend the perturbative treatment to sufficiently short scales. The situation will be different for emission line galaxies (ELG), which are the main targets for the upcoming surveys such as DESI [10] or Euclid [9]. The clustering properties of ELGs have recently been measured for the first time by the eBOSS survey [74]. Crucially, these measurements already show that the ELG sample is less affected by the fingers-of-God, and hence one can expect some improvements in cosmological constraints over the LRG-based analysis, due to larger effective $k_{\max }$.

Our analysis can be extended in various ways. First, the theoretical error approach can be applied to the bispectrum data [28]. Second, it would be curious to check to what extent it can be useful for the ELG sample. Third, our formalism can be extended to the case of the projected statistics such as weak lensing, where it could play an important role to minimize systematic biases due to imperfect theoretical modeling; see e.g., [75]. We leave these research directions for future work.

\section{ACKNOWLEDGMENTS}

We thank Roman Scoccimarro for sharing with us the power spectra from the Las amas $N$-body simulation. We are grateful to Marcel Schmittfull and Matias Zaldarriaga for valuable discussions. We thank Oliver Philcox and Martin White for their valuable comments on the draft. A. C. and M. I. are supported by the RFBR Grant No. 20-02-00982. All numerical calculations were performed with the HybriLIT heterogeneous computing platform (LIT, JINR) [76].

\section{APPENDIX A: THEORETICAL ERROR ENVELOPE}

\section{Comparison to perturbation theory}

The key ingredients of the theoretical error approach are the envelope for the theoretical error covariance $E(k)$ and the theoretical error mean $\bar{P}^{(\mathrm{TE})}(k)$. By definition the theoretical error is the difference between the full model describing the data and an analytic approximation to that model. This suggests that a reasonable choice for the mean should be a 

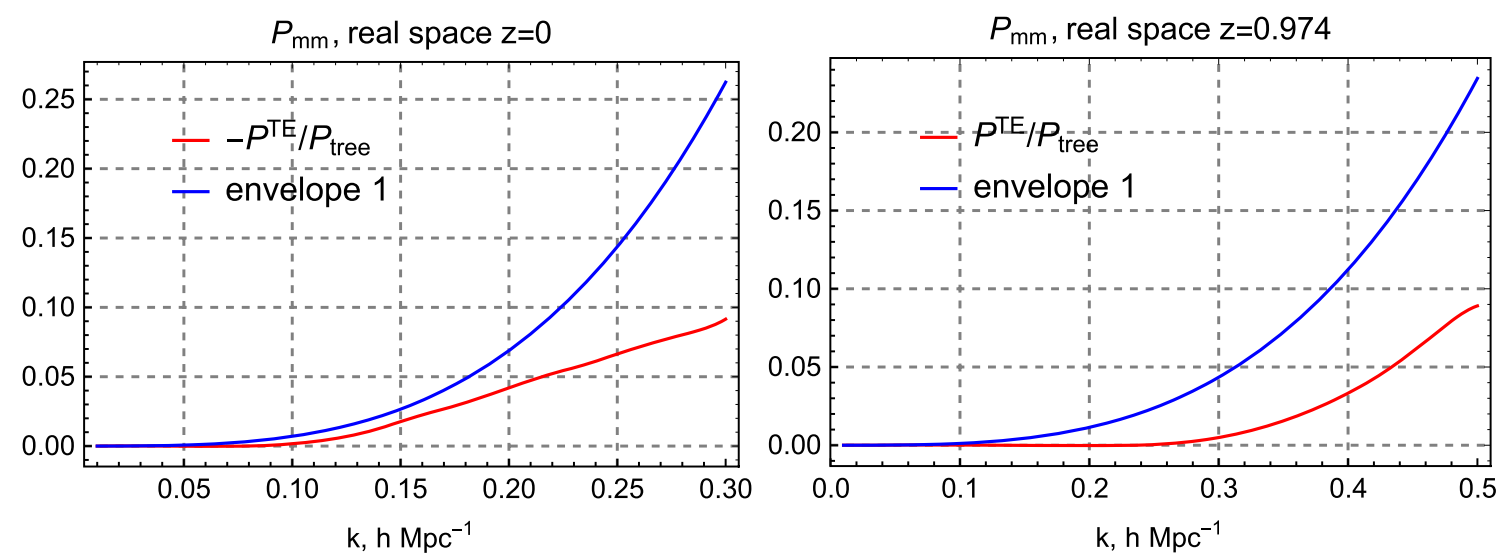

FIG. 13. Theoretical error envelopes for dark matter in real space, normalized to the tree-level spectra. We show the envelope suggested by Ref. [28] (envelope 1) and the envelope $\bar{P}^{(\mathrm{TE})}$ measured directly from the data.

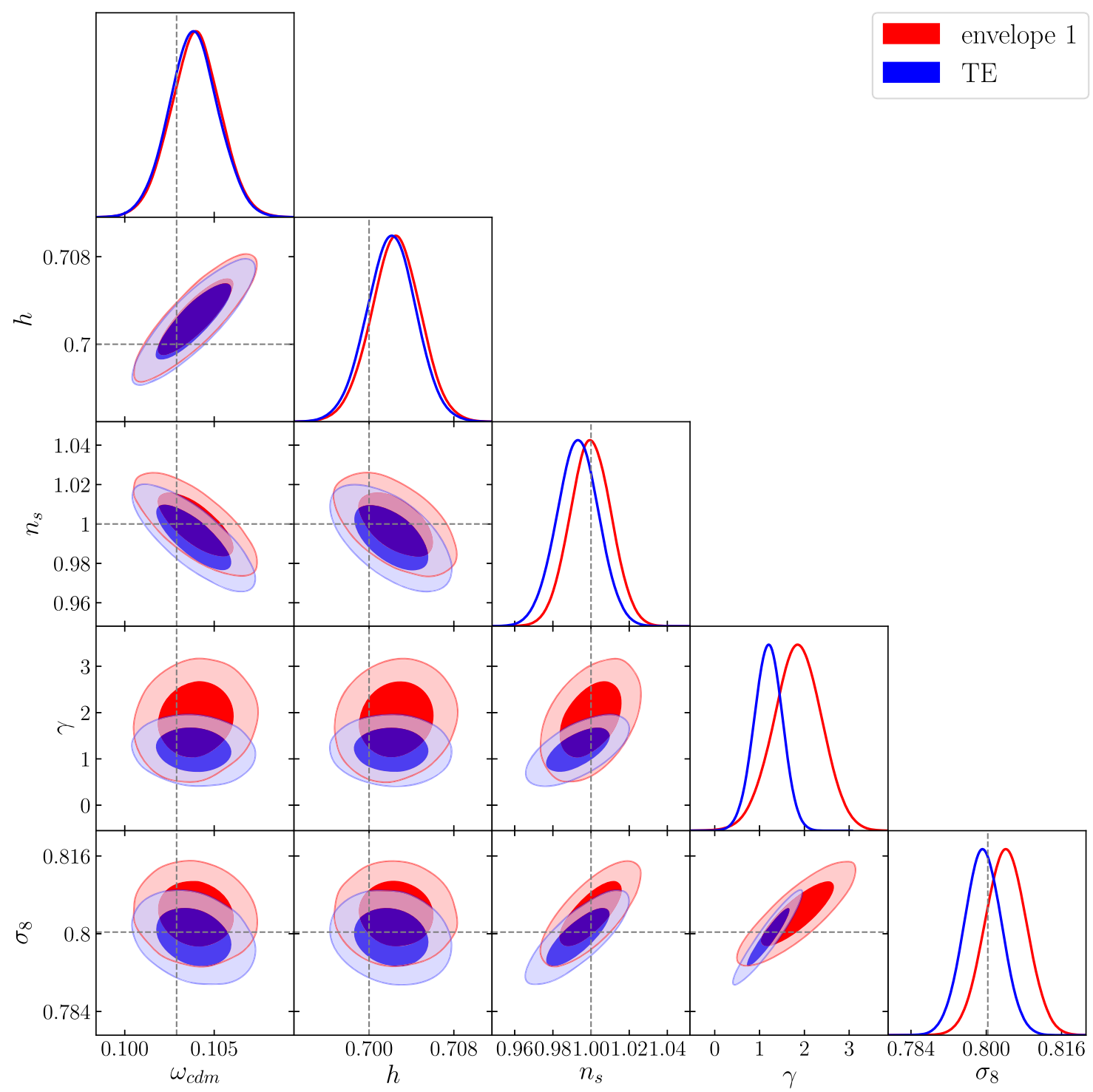

FIG. 14. Triangle plot with cosmological constraints from the real space dark matter power spectrum at $z=0$ for two choices of the theoretical error likelihood. 
residual between some simulation result (or the data) and the approximate model fitted to some sufficiently low $k_{\max }^{\mathrm{fid}}$. The theoretical error covariance is then used for $k>k_{\max }^{\mathrm{fid}}$.

As a second step, we must define the relationship between $E$ and $\bar{P}^{(\mathrm{TE})}$. The toy model of the theoretical error entirely given by a higher-order counterterm suggests that the mean and the envelope should have the same order of magnitude. We impose an even stronger relation

$$
E(k, z)=\bar{P}^{(\mathrm{TE})}(k, z)
$$

such that the theoretical error likelihood is characterized by a single shape. The original work [28] assumed the zero mean and the following envelope:

$$
\begin{aligned}
\text { envelope 1: } E(k . z)= & D^{6}(z)\left(\frac{k}{0.45 h \mathrm{Mpc}^{-1}}\right)^{3.3} \\
& \times P_{\operatorname{lin}}(k, z=0),
\end{aligned}
$$

where $D(z)$ is the linear growth factor. This envelope is a smooth fit to the two-loop dark matter power spectrum computed in SPT [61], after subtracting the leading UV part. It should be stressed that this two-loop SPT correction still has a strong residual unphysical sensitivity to the ultraviolet modes at the subleading order [77-79], and hence the actual effective field theory result is smaller than the SPT prediction. Nevertheless, we expect Eq. (A2) to be order-of-magnitude accurate. In Fig. 13 we compare the prediction of Eq. (A2) to the envelope that we extracted from the data. We used $k_{\max }^{\mathrm{fid}}=0.1 \mathrm{hMpc}^{-1}$ for $z=0$ and $k_{\max }^{\mathrm{fid}}=0.26 h \mathrm{Mpc}^{-1}$ for $z=0.974$. Indeed, as expected, we can see that Eq. (A2) and our envelope (A1) agree by order of magnitude, but Eq. (A2) systematically overestimates the difference between the data and the prediction of one-loop perturbation theory by a factor of $\sim 3$.

It is instructive to compare the two different theoretical error prescriptions at the level of the cosmological
TABLE V. The marginalized 1D intervals for the cosmological parameters estimated from the Las Damas real space dark matter power spectra at $z=0$ for two different theoretical error prescriptions. The table contains fitted parameters (first column), fiducial values used in simulations (second column), the results for the envelope 1 template with $\bar{P}^{(\mathrm{TE})}=0$ (third column), and that for the baseline theoretical error analysis TE (fourth column). $\gamma$ is quoted in units $\left[h^{-1} \mathrm{Mpc}\right]^{2}$.

\begin{tabular}{lccc}
\hline \hline Par & Fid & Envelope 1 & TE \\
\hline$\omega_{\text {cdm }}$ & 0.1029 & $0.1040_{-1.4 \times 10^{-3}}^{+1.4 \times 10^{-3}}$ & $0.1039_{-1.5 \times 10^{-3}}^{+1.3 \times 10^{-3}}$ \\
$h$ & 0.7 & $0.7025_{-2.3 \times 10^{-3}}^{+2.4 \times 1}$ & $0.7021_{-2.4 \times 10^{-3}}^{+2.4 \times 10^{-3}}$ \\
$n_{s}$ & 1 & $0.9998_{-0.011}^{+0.011}$ & $0.9930_{-0.011}^{+0.011}$ \\
$A$ & 1 & $0.9950_{-0.016}^{+0.015}$ & $0.9897_{-0.016}^{+0.016}$ \\
$\Omega_{m}$ & 0.25 & $0.2504_{-1.7 \times 10^{-3}}^{+1.7 \times 1.3}$ & $0.2504_{-1.7 \times 10^{-3}}^{+1.6 \times 10^{-3}}$ \\
$\sigma_{8}$ & 0.8003 & $0.8041_{-4.5 \times 10^{-3}}^{+4.5}$ & $0.7992_{-4.1 \times 10^{-3}}^{+4.1 \times 10^{-3}}$ \\
$\gamma$ & $\ldots$ & $1.85_{-0.53}^{+0.56}$ & $1.19_{-0.32}^{+0.33}$ \\
\hline \hline
\end{tabular}

constraints. To that end we have run the cosmological analysis of the real space dark matter power spectrum at $z=0$ with $\bar{P}^{(\mathrm{TE})}=0$ and the envelope Eq. (A2). The resulting 2D posteriors are shown in Fig. 14, whereas 1D marginalized constraints are listed in Table V. One can see that only the measurements of $\sigma_{8}$ and the effective sound speed are significantly affected. This shows that the use of the perturbation theory-inspired templates can overestimate the actual error bars on the amplitude parameters. However, the shape and distance parameters $\omega_{\mathrm{cdm}}, n_{s}, h$ are expected to be less affected by this choice. A similar situation was found in Ref. [5], which showed that the BAO measurements using the theoretical error covariance do not depend on the exact shape of the theoretical error envelope.

The situation becomes more complicated for redshift space multipoles, where the complete two-loop calculation has not yet been done. Thus, even the perturbation theory estimates can be very uncertain. One can consider two possible estimates,
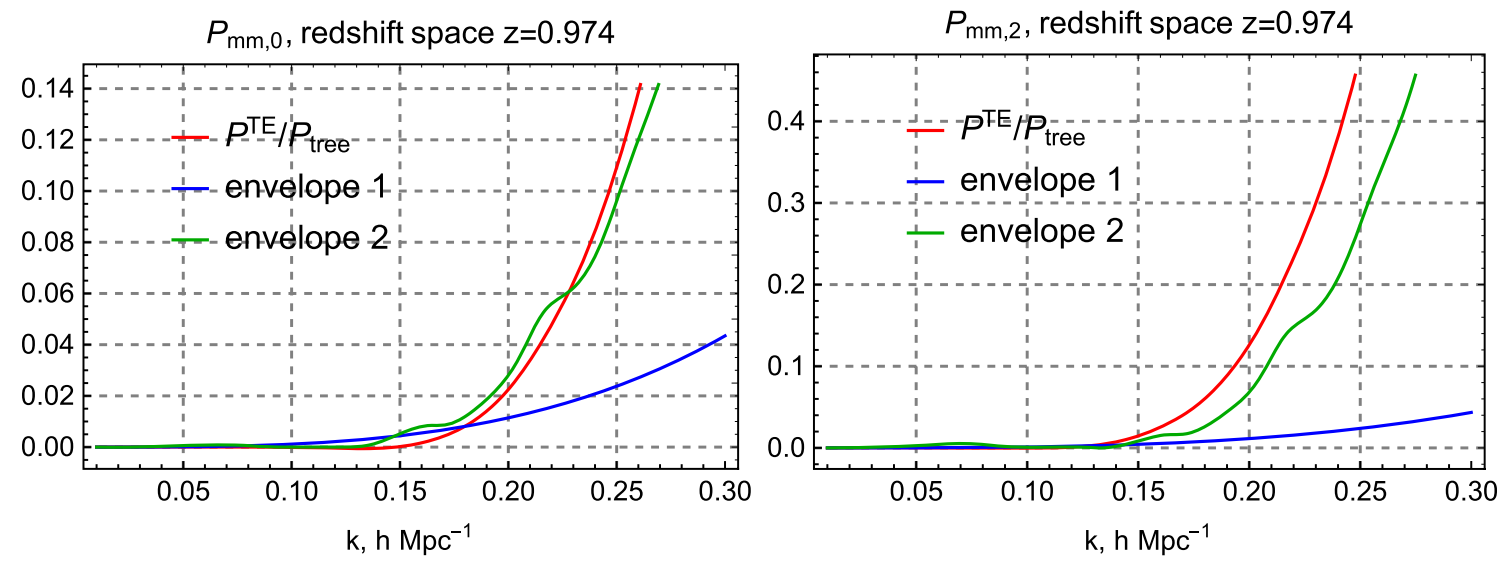

FIG. 15. Theoretical error envelopes for the monopole (left panel) and quadrupole (right panel) moments of the power spectrum of dark matter in redshift space, normalized to the tree-level spectra. See Eq. (A3) for the description of the estimates. 

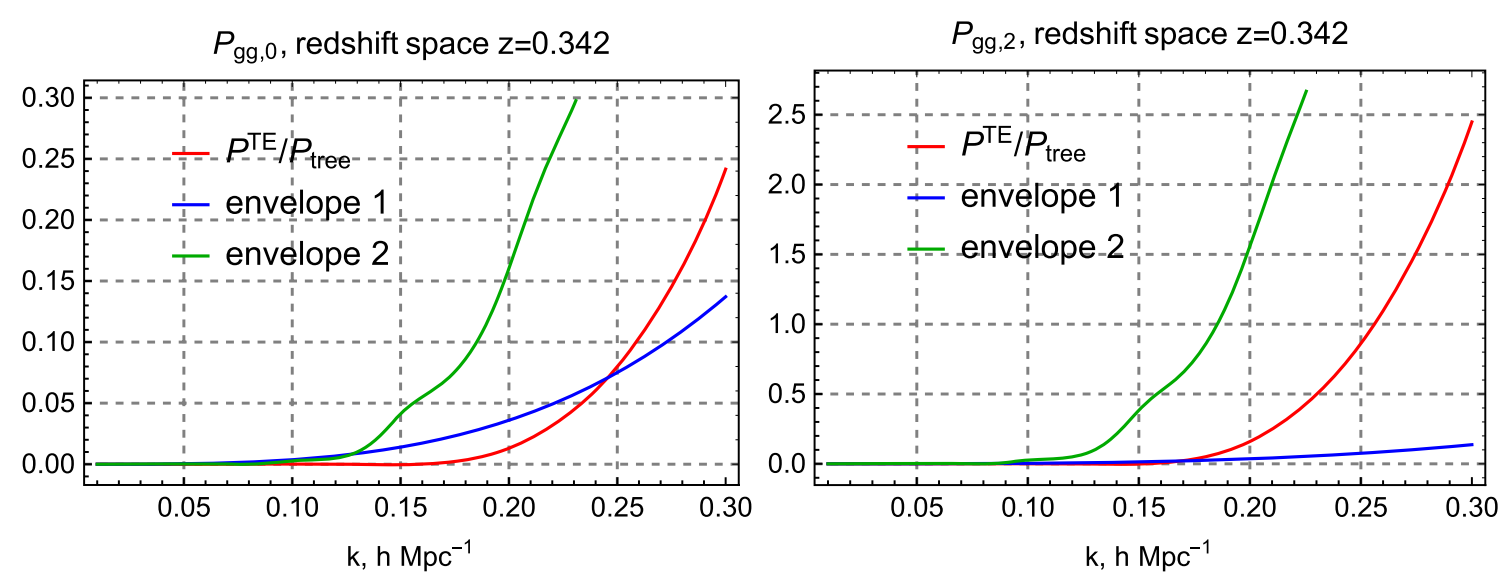

FIG. 16. Same as Fig. 15 but for galaxies in redshift space.

envelope 1: $E_{\ell}(k, z)=D^{6}(z)\left(\frac{k}{0.45 h \mathrm{Mpc}^{-1}}\right)^{3.3} P_{\ell, \text { tree }}(k, z)$, envelope 2: $E_{\ell}(k, z)=\frac{P_{\ell, 1-\text { loop }}^{2}(k, z)}{P_{\ell, \text { tree }}(k, z)}$.

The envelope 2 is based on the disconnected two-loop diagram which involves a product of two one-loop diagrams, but without an extra propagator $P_{\ell \text {,tree }}$.

Comparing these two estimates with our baseline choice (at $k_{\max }^{\mathrm{fid}}=0.12 \mathrm{hMpc}^{-1}$ ) in Fig. 15, one sees that "envelope 2" describes the actual difference between the one-loop PT model and the data surprisingly well. In contrast, "envelope 1" underestimates the theoretical error quite significantly, by more than 1 order of magnitude.

Finally, Fig. 16 shows the envelopes for the redshift space galaxies. Our envelope is computed using $k_{\max }^{\text {fid }}=$ $0.16 \mathrm{~h} \mathrm{Mpc}^{-1}$. We can see that envelope 1 agrees well with our theoretical error for the monopole, while envelope 2 largely overestimates it on short scales. In contrast, envelope 1 significantly underestimates the theoretical error for the quadrupole, whereas envelope 1 still overestimates it; our envelope lies in between these two perturbation theory estimates. All in all, we conclude that the redshift space theoretical error envelopes that we use in this paper roughly agree with the perturbation theory estimates, but the latter are quite uncertain.

\section{Choice of $\boldsymbol{k}_{\max }^{\mathrm{fid}}$}

In this section we argue our choice of $k_{\max }^{\mathrm{fid}}$ in the theoretical error analyses. For that, we fix the cosmological parameters to their fiducial values and find the best-fitting values of nuisance parameters varying $k_{\max }$. Then, we extract the best-fit reduced statistic $\chi^{2} / N_{\text {dof }}\left(N_{\text {dof }}=N_{\text {bins }}-N_{\text {params }}\right)$ for each $k_{\max }$. The results for the real space dark matter are shown in the upper left panel of Fig. 17. Note that the typical values of $\chi^{2} / N_{\text {dof }}$ are around 0.2 , which is a result of using the reduced volume $V=100(\mathrm{Gpc} / h)^{3}$ in the covariance instead of the true cumulative volume of the Las Damas
Oriana simulation $V=553(\mathrm{Gpc} / h)^{3}$. We see that the $\chi^{2}$ profile blows up at $k_{\max }=0.14 h \mathrm{Mpc}^{-1}$ for $z=0$ and at $k_{\max }=0.30 \mathrm{hMpc}^{-1}$ for $z=0.974$. This is an indication that the one-loop perturbation theory model becomes invalid at these scales. Thus, we choose smaller fiducial cuts $k_{\max }^{\mathrm{fid}}$, where the $\chi^{2}$ profile is still flat: $k_{\max }^{\text {fid }}=0.10 h \mathrm{Mpc}^{-1}$ for $z=0$ and $k_{\max }^{\mathrm{fid}}=0.26 \mathrm{hMpc}^{-1}$ for $z=0.974$. Operationally, it is suggestive to use

$$
k_{\max }^{\mathrm{fid}}=k_{2-\text { loop }}-\Delta k^{\prime}, \quad \Delta k^{\prime}=0.04 h \mathrm{Mpc}^{-1} .
$$

The fiducial $k_{\max }$ for other cases are chosen in a completely similar fashion. The results for dark matter in redshfit space are shown in the upper right panel of Fig. 17. We found that the reduced $\chi^{2} / N_{\text {dof }}$ statistics remains flat up to $k_{\max }=0.16 \mathrm{hMpc}^{-1}$. However, in order to be conservative, we choose $k_{\max }^{\mathrm{fid}}=0.12 \mathrm{hMpc}^{-1}$ in our analysis.

The results for the real space galaxies are shown in the lower left panel of Fig. 17. In this case, the picture is not so obvious due to the large shot noise contribution in the covariance, which can be larger than the theoretical error covariance. For this reason, one-loop perturbation theory provides an accurate description of the galaxy power spectrum up to nonlinear scale $k_{\max } \approx 0.3 h \mathrm{Mpc}^{-1}$. Since we do not see any sign of the bias up to $k_{\max }=0.3 \mathrm{hMpc}^{-1}$, we conclude that the theoretical error is negligibly smaller than the statistical covariance dominated by shot noise. The situation is somewhat different for the galaxy-matter cross-spectrum for which the $\chi^{2}$ profile shows some scale dependence for $k_{\max }>0.22 \mathrm{hMpc}^{-1}$. In this case, we choose $k_{\max }^{\mathrm{fid}}=0.18 \mathrm{hMpc}^{-1}$ in our theoretical error analysis of $P_{\mathrm{gm}}$.

Finally, we display the best fit $\chi^{2} / N_{\text {dof }}$ profile for galaxies in redshift space in the lower right panel of Fig. 17. One can see that the $\chi^{2}$ profile blows up at $k_{\max }>0.2 \mathrm{hMpc}^{-1}$. Given this reason, we choose $k_{\max }^{\mathrm{fid}}=0.16 \mathrm{hMpc}^{-1}$. 

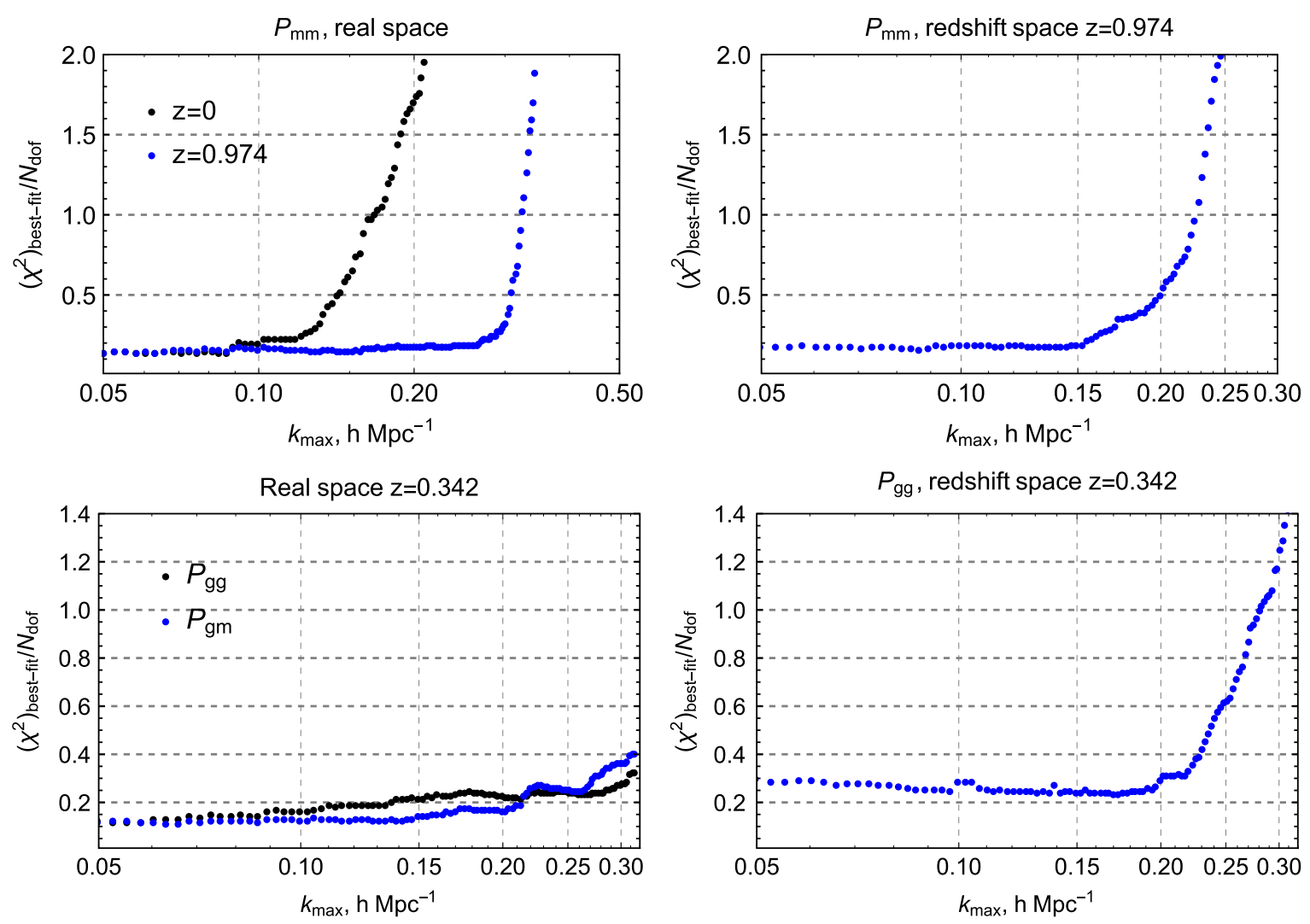

FIG. 17. Best-fit reduced $\chi^{2} / N_{\text {dof }}$ as a function of $k_{\max }$ for various likelihoods considered in this paper.

It should be stressed that using the $\chi^{2}\left(k_{\max }\right)$ profile is inappropriate for defining the baseline cut of the $k_{\max }$ analysis because the fit can be biased at $k_{\max }$ lower than $k_{2-\text { loop }}$ when the cosmological parameters are varied. Indeed, we see that $k_{2 \text {-loop }}$ is typically larger than the baseline cuts $k_{\max }$ in our main analyses. Hence, using the $\chi^{2}$ profile is appropriate only for the theoretical error and not to fix the baseline $k_{\max }$.

\section{APPENDIX B: SUPPLEMENTARY MATERIAL ON THE FINGERS-OF-GOD MODELING}

We show the 1D marginalized limits on the parameters of different FoG models from the redshift space spectrum analyses for $z=0$ in Table VI and for $z=0.974$ in Table VII.

Figure 18 displays the parameter constraints for different FoG models including "EFT + FoG," which are

TABLE VI. The marginalized 1D intervals for the amplitude and nuisance parameters estimated from the monopole and quadrupole moments of the Las Damas dark matter redshift space power spectrum at $z=0$ for fixed fiducial cosmology. We show the fitted parameters (first column), the fiducial values used in simulations (second column), and the resulting parameter constraints in different models: $(\Sigma)$ (third column), $\left(c_{0}, c_{2}\right)$ (fourth column), and $\left(c_{0}, c_{2}, b_{4}\right)$ (fifth column) at $k_{\max }=0.1 \mathrm{~h} / \mathrm{Mpc} . c_{0}, c_{2}, b_{4}$ are quoted in units $\left[h^{-1} \mathrm{Mpc}\right]^{2},\left[h^{-1} \mathrm{Mpc}\right]^{2}$, $\left[h^{-1} \mathrm{Mpc}\right]^{4}$, respectively.

\begin{tabular}{lcccc}
\hline \hline Par & & \multicolumn{3}{c}{$z=0$} \\
\cline { 3 - 5 } & Fid & $(\Sigma)$ & $\left(c_{0}, c_{2}\right)$ & $\left(c_{0}, c_{2}, b_{4}\right)$ \\
\hline$A$ & 1 & $0.9873_{-1.5 \times 10^{-3}}^{+1.5 \times 10^{-3}}$ & $1.0063_{-2.9 \times 10^{-3}}^{+2.9 \times 10^{-3}}$ & $1.0031_{-3.3 \times 10^{-3}}^{+3.3 \times 10^{-3}}$ \\
$\sigma_{8}$ & 0.8003 & $0.7952_{-6.0 \times 10^{-4}}^{+6.0 \times 10^{-4}}$ & $0.8028_{-1.2 \times 10^{-3}}^{+1.2 \times 10^{-3}}$ & $0.8015_{-1.3 \times 10^{-3}}^{+1.3 \times 10^{-3}}$ \\
$\Sigma$ & $\ldots$ & $7.75_{-0.15}^{+0.15}$ & $\cdots$ & $\cdots$ \\
$c_{0}$ & $\ldots$ & $\ldots$ & $5.30_{-0.27}^{+0.28}$ & $4.19_{-0.60}^{+0.60}$ \\
$c_{2}$ & $\ldots$ & $\ldots$ & $25.71_{-0.95}^{+0.94}$ & $17.12_{-4.21}^{+4.30}$ \\
$10^{-3} b_{4}$ & $\ldots$ & $\ldots$ & $\cdots$ & $12.63_{-6.14}^{+5.99}$ \\
\hline \hline
\end{tabular}


TABLE VII. The marginalized 1D intervals for the amplitude and nuisance parameters estimated from the monopole and quadrupole moments of the Las Damas dark matter redshift space power spectrum at $z=0.974$ for fixed fiducial cosmology. We show the fitted parameters (first column), and the resulting parameter constraints in different models: $(\Sigma)$ (second column), $\left(c_{0}, c_{2}\right)$ (third column) at $k_{\max }=0.1 \mathrm{~h} / \mathrm{Mpc}$ and $\left(\Sigma, c_{0}\right)$ (fourth column), $\left(c_{0}, c_{2}\right)$ (fifth column), $\left(c_{0}, c_{2}, b_{4}\right)$ (sixth column) at $k_{\max }=0.14 \mathrm{~h} / \mathrm{Mpc} . c_{0}, c_{2}, b_{4}$ are quoted in units $\left[h^{-1} \mathrm{Mpc}\right]^{2}$, $\left[h^{-1} \mathrm{Mpc}\right]^{2},\left[h^{-1} \mathrm{Mpc}\right]^{4}$, respectively.

\begin{tabular}{lccccc}
\hline \hline & \multicolumn{5}{c}{$z=0.974$} \\
\cline { 2 - 5 } & \multicolumn{4}{c}{$k_{\max }=0.1 h / \mathrm{Mpc}$} & \multicolumn{3}{c}{$k_{\max }=0.14 h / \mathrm{Mpc}$} \\
\hline & $(\Sigma)$ & $\left(c_{0}, c_{2}\right)$ & $\left(\Sigma, c_{0}\right)$ & $\left(c_{0}, c_{2}\right)$ & $\left(c_{0}, c_{2}, b_{4}\right)$ \\
$\sigma_{8}$ & $0.9936_{-1.5 \times 10^{-3}}^{+1.5 \times 10^{-3}}$ & $1.0021_{-2.8 \times 10^{-3}}^{+2.8 \times 10^{-3}}$ & $1.0046_{-1.7 \times 10^{-3}}^{+1.7 \times}$ & $1.0052_{-1.8 \times 10^{-3}}^{+1.8 \times 10^{-3}}$ & $1.0017_{-2.0 \times 10^{-3}}^{+1.9 \times 10^{-3}}$ \\
$\Sigma$ & $0.7977_{-6.1 \times 10^{-4}}^{+6.1 \times 10^{-4}}$ & $0.8011_{-1.1 \times 10^{-3}}^{+1.1 \times 10^{-3}}$ & $0.8021_{-6.9 \times 10^{-4}}^{+6.9 \times 10^{-4}}$ & $0.8024_{-7.0 \times 10^{-4}}^{+7.0 \times 10^{-4}}$ & $0.8010_{-7.9 \times 10^{-4}}^{+7.8 \times 10^{-4}}$ \\
$c_{0}$ & $3.30_{-0.11}^{+0.11}$ & $\ldots$ & $3.60_{-0.03}^{+0.03}$ & $\ldots$ & $\cdots$ \\
$c_{2}$ & $\ldots$ & $3.82_{-0.34}^{+0.34}$ & $0.94_{-0.08}^{+0.08}$ & $4.31_{-0.11}^{+0.11}$ & $3.41_{-0.26}^{+0.26}$ \\
$10^{-3} b_{4}$ & $\ldots$ & $13.14_{-0.82}^{+0.83}$ & $\cdots$ & $15.19_{-0.30}^{+0.30}$ & $10.88_{-1.13}^{+1.15}$ \\
\hline \hline
\end{tabular}

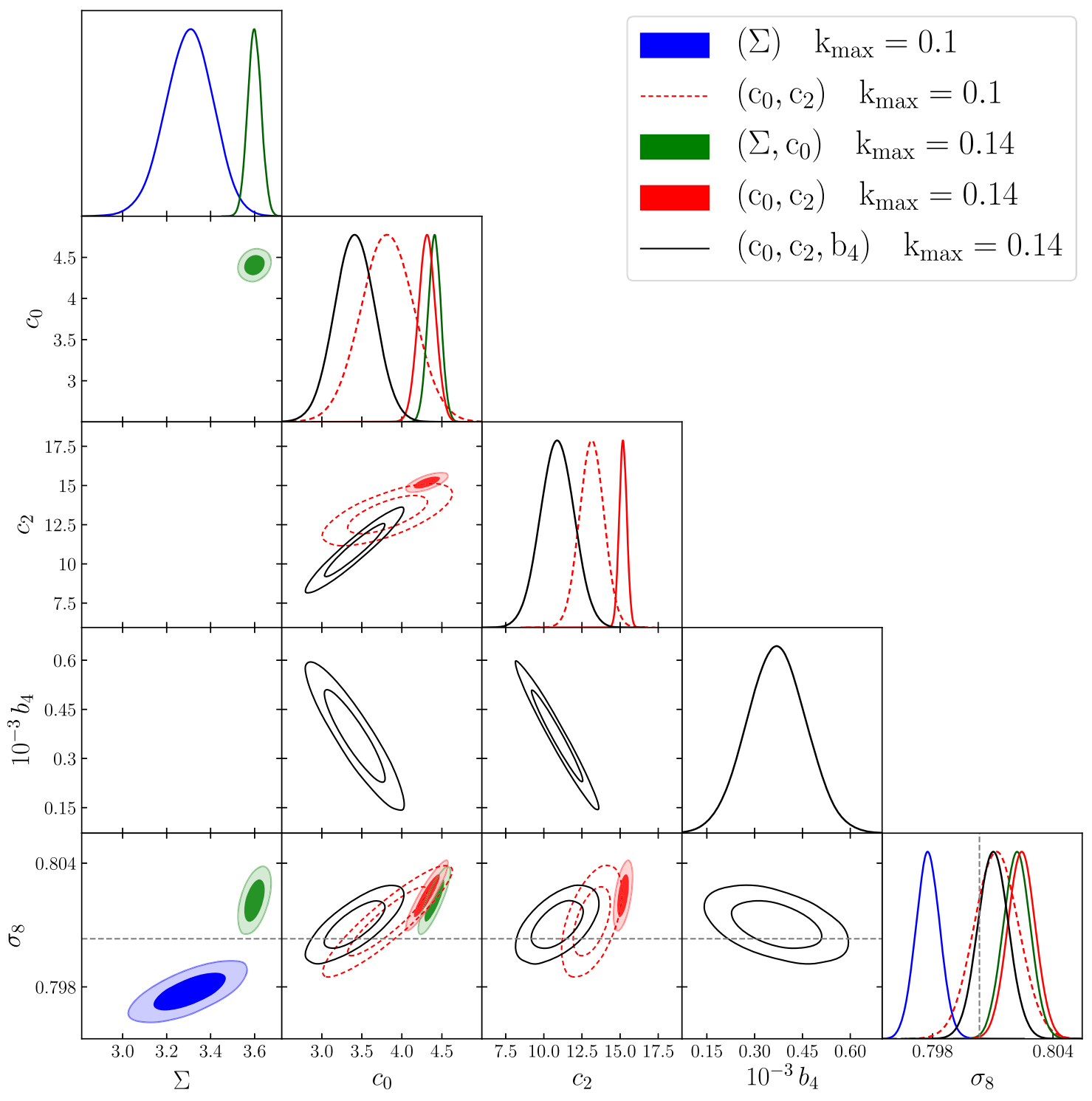

FIG. 18. Same as Fig. 6, but with the additional EFT + FoG model (5.8), characterized by the parameters $\left(c_{0}, \Sigma\right)$. 
TABLE VIII. The marginalized 1D intervals for the cosmological parameters estimated from the Las Damas real space dark matter power spectra at $z=0$. The table contains fitted parameters (first column), fiducial values used in simulations (second column), the results of the baseline $k_{\max }$ analysis (third column), and outcomes of TE approaches with default (fourth column) and new fiducial cosmology (fifth column) termed TE 2. $\gamma$ is quoted in units $\left[h^{-1} \mathrm{Mpc}\right]^{2}$.

\begin{tabular}{lcccc}
\hline \hline & & \multicolumn{3}{c}{$z=0$} \\
\cline { 3 - 5 } Par & Fid & $k_{\max }=0.12$ & TE & TE 2 \\
\hline$\omega_{\mathrm{cdm}}$ & 0.1029 & $0.1023_{-1.8 \times 10^{-3}}^{+1.6 \times 10^{-3}}$ & $0.1039_{-1.5 \times 10^{-3}}^{+1.4 \times 10^{-3}}$ & $0.1036_{-1.4 \times 10^{-3}}^{+1.4 \times 10^{-3}}$ \\
$h$ & 0.7 & $0.6983_{-3.4 \times 10^{-3}}^{+3.3 \times 10^{-3}}$ & $0.7021_{-2.4 \times 10^{-3}}^{+2.4}$ & $0.7018_{-2.3 \times 10^{-3}}^{+2.3 \times 10^{-3}}$ \\
$n_{s}$ & 1 & $1.008_{-0.013}^{+0.014}$ & $0.9930_{-0.011}^{+0.011}$ & $0.9981_{-0.010}^{+0.010}$ \\
$A$ & 1 & $1.014_{-0.020}^{+0.020}$ & $0.9897_{-0.016}^{+0.016}$ & $0.9952_{-0.015}^{+0.015}$ \\
$\Omega_{m}$ & 0.25 & $0.2499_{-2.3 \times 10^{-3}}^{+2.2 \times 10^{-3}}$ & $0.2504_{-1.7 \times 10^{-3}}^{+1.6 \times 15}$ & $0.2502_{-1.7 \times 10^{-3}}^{+1.6 \times 10^{-3}}$ \\
$\sigma_{8}$ & 0.8003 & $0.8044_{-4.5 \times 10^{-3}}^{+4.5 \times 10^{-3}}$ & $0.7992_{-4.1 \times 10^{-3}}^{+4.1 \times 1}$ & $0.8018_{-3.2 \times 10^{-4}}^{+3.2 \times 10^{-4}}$ \\
$\gamma$ & $\cdots$ & $1.60_{-0.31}^{+0.33}$ & $1.19_{-0.32}^{+0.33}$ & $1.41_{-0.22}^{+0.22}$ \\
\hline \hline
\end{tabular}

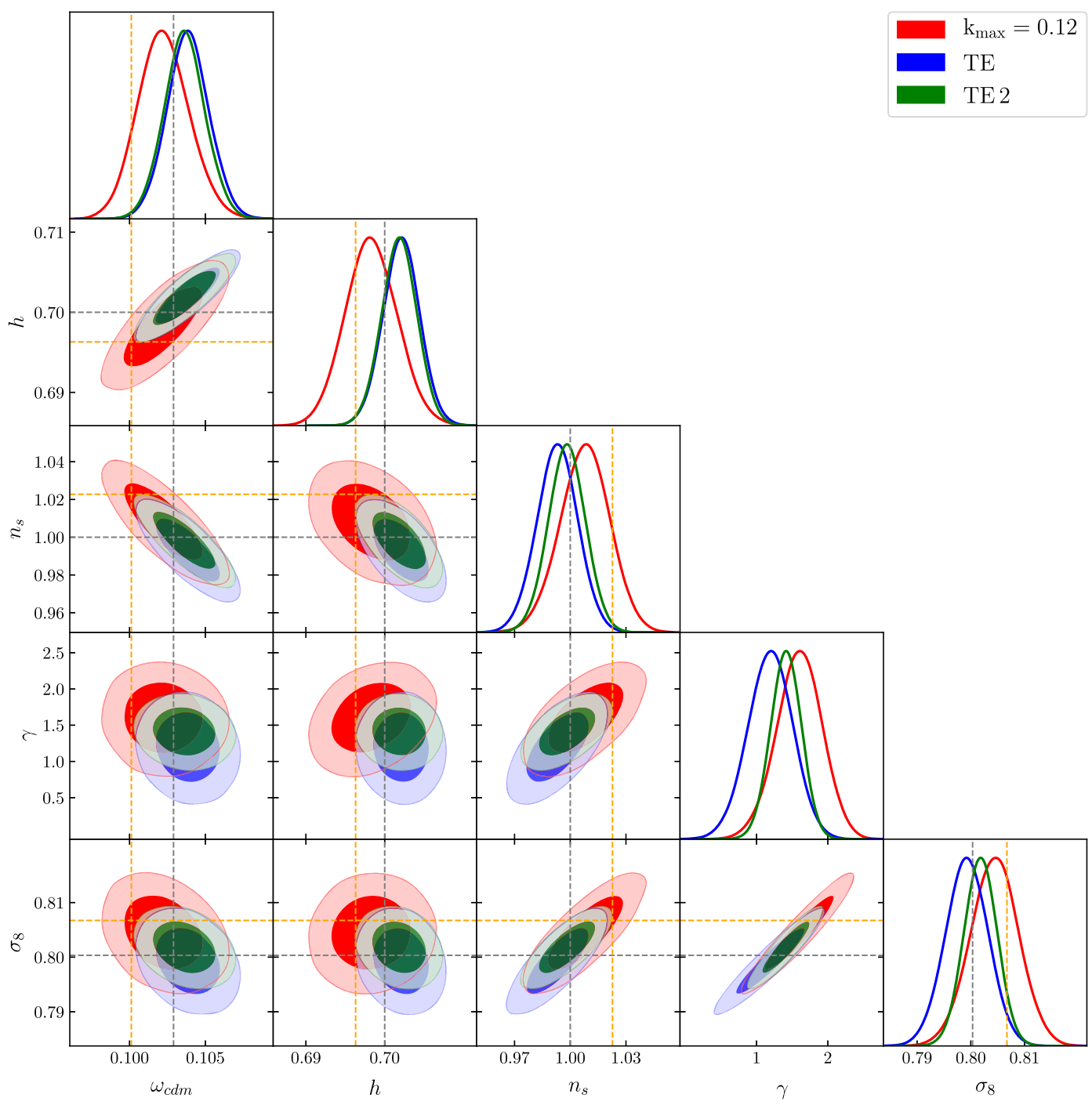

FIG. 19. Triangle plot for the cosmological and nuisance parameters measured from the real space dark matter power spectrum of the Las Damas simulations at $z=0$ for the $k_{\max }$ analysis and two choices of the fiducial cosmology in the TE approach. The dashed orange lines mark the fiducial cosmological parameters used in the TE 2 analysis, whereas the gray dashed lines mark the fiducial cosmology used in the baseline analysis. It coincides with the true mock cosmology. 
extracted from the matter redshift space power spectrum at $z=0.974$.

\section{APPENDIX C: DEPENDENCE ON FIDUCIAL COSMOLOGY}

In our algorithm, the theory prediction for the TE mean has been calculated using the true cosmology of mock catalogs. As the actual cosmological parameters are a priori unknown, we need to check whether our results depend on the choice of the fiducial cosmology. To estimate the corresponding uncertainty we repeat the TE analysis with a different fiducial cosmology. To that end we use a set of cosmological parameters from a randomly chosen step from MCMC chains of the baseline $k_{\max }$ analyses for dark matter and galaxies in real and redshift spaces. In each of these cases, we require that this new parameter set deviates noticeably from the true mock cosmology, but still stays within the 95\% C.L. Then, for this chosen cosmology we calculate the TE mean following the algorithm described in the main text. We will fit the real space dark matter $(z=0)$, redshift space dark matter $(z=0.974)$, and redshift space galaxies $(z=0.342)$ mock data and compare the resulting posterior distribution with the results of Secs. IV, V, and VII.

Let us begin with the real space dark matter $(z=0)$. The marginalized 1D parameter constraints are listed in Table VIII (fifth column). The 2D posterior distributions are shown in Fig. 19 (green contours). We have found that posterior distributions for all parameters except for $\sigma_{8}, n_{s}$, and $\gamma$ are not altered by the different choice of the fiducial cosmology. However, the error bars on $\sigma_{8}, n_{s}$, and $\gamma$ decrease by $20 \%, 10 \%$, and $30 \%$, respectively, compared to the baseline TE analysis. These changes can be readily understood. First, $\sigma_{8}$ and $n_{s}$ control the amplitude of the theoretical error envelope $E$. It implies that the uncertainty in the choice of fiducial cosmology for $E$ propagates into uncertainty for $\sigma_{8}$ and $n_{s}$ measurements. Second, $\sigma_{8}$ and $\gamma$ are strongly degenerate, and hence the aforementioned

TABLE IX. The marginalized 1D intervals for the cosmological and nuisance parameters estimated from the monopole and quadrupole moments of the Las Damas dark matter redshift space power spectrum at $z=0.974$. We show the fitted parameters (first column), fiducial values used in simulations (second column), and the resulting parameter constraints for the baseline $k_{\max }$ analysis (third column) and the theoretical error approach with default (fourth column) and new fiducial cosmology (fifth column) termed TE 2. $c_{0}, c_{2}, b_{4}$ are quoted in units $\left[h^{-1} \mathrm{Mpc}\right]^{2}$, $\left[h^{-1} \mathrm{Mpc}\right]^{2},\left[h^{-1} \mathrm{Mpc}\right]^{4}$, respectively.

\begin{tabular}{|c|c|c|c|c|}
\hline \multirow[b]{2}{*}{ Par } & \multirow[b]{2}{*}{ Fid } & \multicolumn{3}{|c|}{$z=0.974$} \\
\hline & & $k_{\max }=0.14 h / \mathrm{Mpc}$ & $\mathrm{TE}$ & $\mathrm{TE} 2$ \\
\hline$\omega_{\mathrm{cdm}}$ & 0.1029 & $0.1023_{-1.5 \times 10^{-3}}^{+1.4 \times 10^{-3}}$ & $0.1026_{-1.0 \times 10^{-3}}^{+1.1 \times 10^{-3}}$ & $0.1011_{-1.0 \times 10^{-3}}^{+1.1 \times 10^{-3}}$ \\
\hline$h$ & 0.7 & $0.6993_{-2.6 \times 10^{-3}}^{+2.4 \times 10^{-3}}$ & $0.6995_{-1.6 \times 10^{-3}}^{+1.0 \times 10^{-3}}$ & $0.6969_{-1.6 \times 10^{-3}}^{+1.6 \times 10^{-3}}$ \\
\hline$n_{s}$ & 1 & $1.0083_{-0.011}^{+0.012}$ & $1.0028_{-8.5 \times 10^{-3}}^{+8.5 \times 10^{-3}}$ & $1.0149_{-7.7 \times 10^{-3}}^{+7.6 \times 10^{-3}}$ \\
\hline$A$ & 1 & $1.0096_{-0.016}^{+0.016}$ & $1.0043_{-0.011}^{+0.010}$ & $1.0221_{-0.011}^{+0.010}$ \\
\hline$\Omega_{m}$ & 0.25 & $0.2493_{-1.8 \times 10^{-3}}^{+1.7 \times 10^{-3}}$ & $0.2498_{-1.3 \times 10^{-3}}^{+1.4 \times 10^{-3}}$ & $0.2486_{-1.3 \times 10^{-3}}^{+1.3 \times 10^{-3}}$ \\
\hline$\sigma_{8}$ & 0.8003 & $0.8033_{-3.1 \times 10^{-3}}^{+3.1 \times 10^{-3}}$ & $0.8012_{-2.8 \times 10^{-3}}^{+2.8 \times 10^{-3}}$ & $0.8036_{-2.1 \times 10^{-3}}^{+2.1 \times 10^{-3}}$ \\
\hline$c_{0}$ & $\cdots$ & $3.66_{-0.37}^{+0.39}$ & $3.29_{-0.39}^{+0.40}$ & $3.51_{-0.19}^{+0.19}$ \\
\hline$c_{2}$ & $\cdots$ & $11.26_{-1.25}^{+1.32}$ & $10.52_{-1.10}^{+1.11}$ & $10.62_{-0.50}^{+0.50}$ \\
\hline $10^{-3} b_{4}$ & $\cdots$ & $0.34_{-0.11}^{+0.10}$ & $0.41_{-0.08}^{+0.108}$ & $0.40_{-0.03}^{+0.03}$ \\
\hline
\end{tabular}




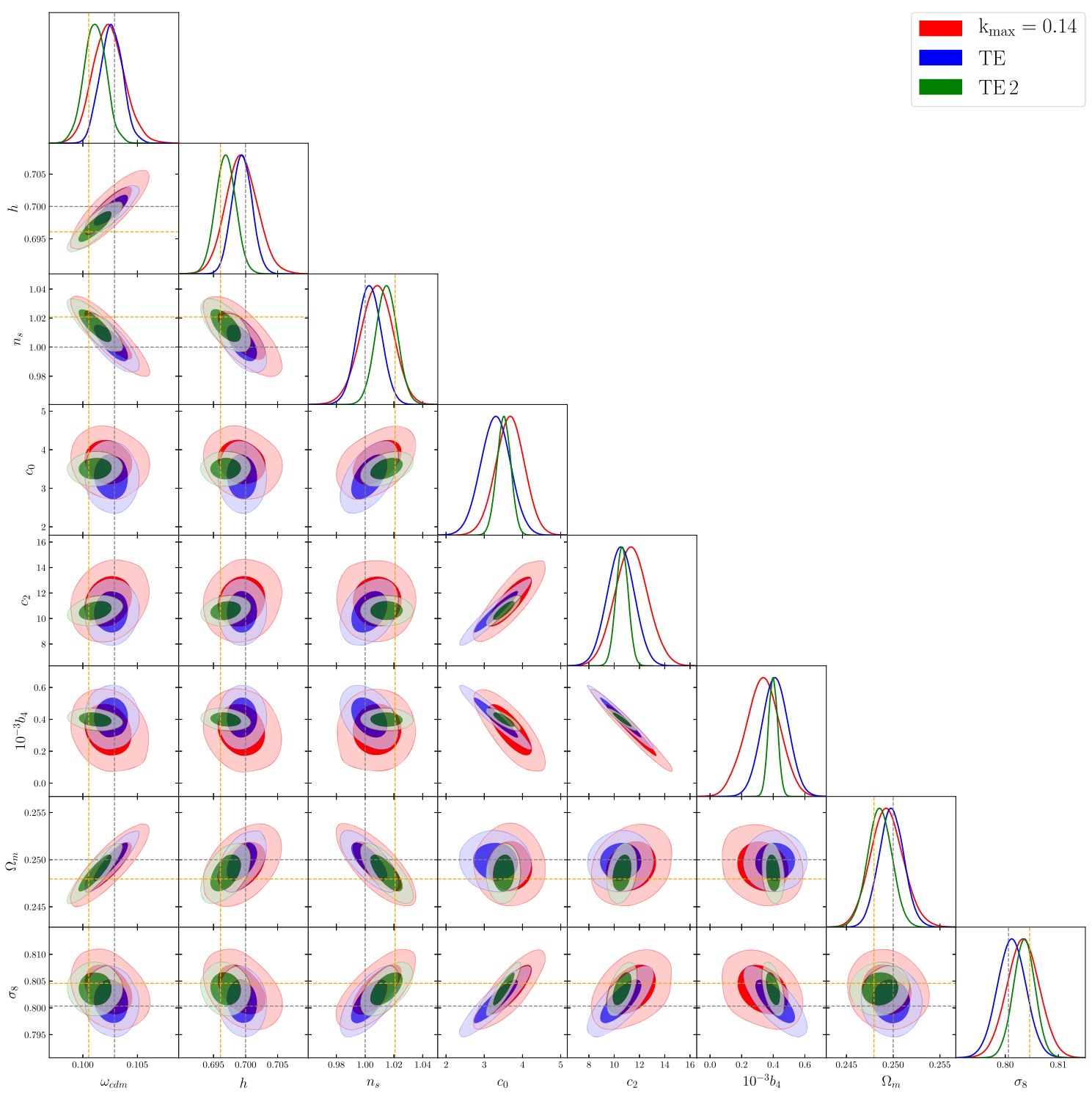

FIG. 20. Triangle plot for the cosmological and nuisance parameters measured from the redshift space dark matter power spectrum of the Las Damas simulations at $z=0.974$ for the $k_{\max }$ analysis and two choices of the fiducial cosmology in the TE approach. $c_{0}, c_{2}, b_{4}$ are quoted in units $\left[h^{-1} \mathrm{Mpc}\right]^{2},\left[h^{-1} \mathrm{Mpc}\right]^{2},\left[h^{-1} \mathrm{Mpc}\right]^{4}$, respectively. Crossing of the dashed orange lines outlines the fiducial cosmological parameters used in the TE 2 analysis. 
uncertainty propagates into the $\gamma$ constraint and worsens it as well.

Now let us consider the case of redshift space dark matter $(z=0.974)$. The marginalized 1D parameter constraints are listed in Table IX (fifth column). The 2D posterior distributions are shown in Fig. 20 (green contours). Just as in real space, changing the fiducial cosmology does not impact uncertainties on $\omega_{\text {cdm }}$ and $h$. However, the error bars on $\sigma_{8}$ and $n_{s}$ decrease by $25 \%$ and $10 \%$. The impact on the counterterms is even stronger. Namely, the constraints on $c_{0}, c_{2}, b_{4}$ become twice weaker compared to those which we have obtained in the baseline TE analysis. We have also found that the posterior distributions are shifted toward the new fiducial cosmology. Nevertheless, the new 2D posteriors enclose the true mock parameters within $95 \%$ C.L. as shown in Fig. 20.

Finally, we discuss redshift space galaxies $(z=0.342)$. The marginalized 1D parameter constraints are listed in
Table X (fifth column). The 2D posterior distributions are shown in Fig. 21 (green contours). In this case, we do not find any significant difference in posterior distributions of the cosmological parameters due to the change of the fiducial cosmology.

Our analysis suggests the following conclusions. The improvement that we have found for dark matter in real space and redshift space on $\sigma_{8}$ and $n_{s}$ parameters may be artificially caused by the specific choice of the fiducial cosmology. However, in the realistic analysis, the TE approach yields robust cosmological constraints which do not depend on the choice of the fiducial cosmology. In this case the TE approach does not provide any information gain in comparison with the analysis with the sharp momentum cut and only serves to optimize the choice of $k_{\max }$. Finally, we would like to point out that we have also studied the convergence of the results with respect to variations of $k_{\max }^{\mathrm{fid}}$ and found statistically indistinguishable results for all considered cases.

TABLE X. The marginalized 1D intervals for the cosmological parameters estimated from the Las Damas redshift space galaxy power spectra at $z=0.342$. The table contains fitted parameters (first column), fiducial values used in simulations (second column), and the results of the baseline $k_{\max }$ analysis (third column) and the outcome of the theoretical error approach with default (fourth column) and new (fifth column) fiducial cosmology. $c_{0}, c_{2}, b_{4}$ are quoted in units $\left[h^{-1} \mathrm{Mpc}\right]^{2},\left[h^{-1} \mathrm{Mpc}\right]^{2},\left[h^{-1} \mathrm{Mpc}\right]^{4}$, respectively.

\begin{tabular}{lcccc}
\hline \hline Par & Fid & $k_{\max }=0.18 h / \mathrm{Mpc}$ & TE & TE 2 \\
\hline$\omega_{\text {cdm }}$ & 0.1029 & $0.1037_{-4.1 \times 10^{-3}}^{+3.5 \times 10^{-3}}$ & $0.1009_{-3.5 \times 10^{-3}}^{+3.5 \times 10^{-3}}$ & $0.1013_{-3.4 \times 10^{-3}}^{+3.4 \times 10^{-3}}$ \\
$h$ & 0.7 & $0.7002_{-5.3 \times 10^{-3}}^{+5.1 \times 10^{-3}}$ & $0.6973_{-5.0 \times 10^{-3}}^{+5.0 \times 10^{-3}}$ & $0.6981_{-4.9 \times 10^{-3}}^{+4.9 \times 3}$ \\
$n_{s}$ & 1 & $0.9909_{-0.026}^{+0.028}$ & $1.0031_{-0.025}^{+0.025}$ & $1.0021_{-0.025}^{+0.025}$ \\
$A$ & 1 & $1.0169_{-0.079}^{+0.073}$ & $1.0571_{-0.081}^{+0.069}$ & $1.0174_{-0.079}^{+0.066}$ \\
$\Omega_{m}$ & 0.25 & $0.2513_{-5.2 \times 10^{-3}}^{+4.6 \times 10^{-3}}$ & $0.2479_{-4.3 \times 10^{-3}}^{+4.3}$ & $0.2480_{-4.4 \times 10^{-3}}^{+4.1 \times 10^{-3}}$ \\
$\sigma_{8}$ & 0.8003 & $0.8069_{-0.022}^{+0.022}$ & $0.8044_{-0.021}^{+0.021}$ & $0.7980_{-0.020}^{+0.021}$ \\
$c_{0}$ & $\ldots$ & $0.77_{-11.48}^{+17.93}$ & $9.40_{-9.68}^{+12.45}$ & $12.88_{-9.41}^{+12.56}$ \\
$c_{2}$ & $\ldots$ & $36.22_{-20.61}^{+28.01}$ & $29.61_{-18.30}^{+23.11}$ & $38.76_{-20.78}^{+25.64}$ \\
$10^{-3} b_{4}$ & $\ldots$ & $1.40_{-0.26}^{+0.21}$ & $1.73_{-0.28}^{+0.25}$ & $1.85_{-0.34}^{+0.31}$ \\
$b_{1}$ & $\ldots$ & $2.17_{-0.07}^{+0.07}$ & $2.16_{-0.07}^{+0.07}$ & $2.19_{-0.07}^{+0.07}$ \\
$b_{2}$ & $\ldots$ & $-0.96_{-0.87}^{+0.64}$ & $-0.92_{-0.78}^{+0.57}$ & $-0.57_{-1.03}^{+0.65}$ \\
$b_{\mathcal{G}_{2}}$ & $\ldots$ & $-0.350_{-0.388}^{+0.364}$ & $-0.330_{-0.368}^{+0.351}$ & $-0.377_{-0.395}^{+0.372}$ \\
\hline \hline
\end{tabular}




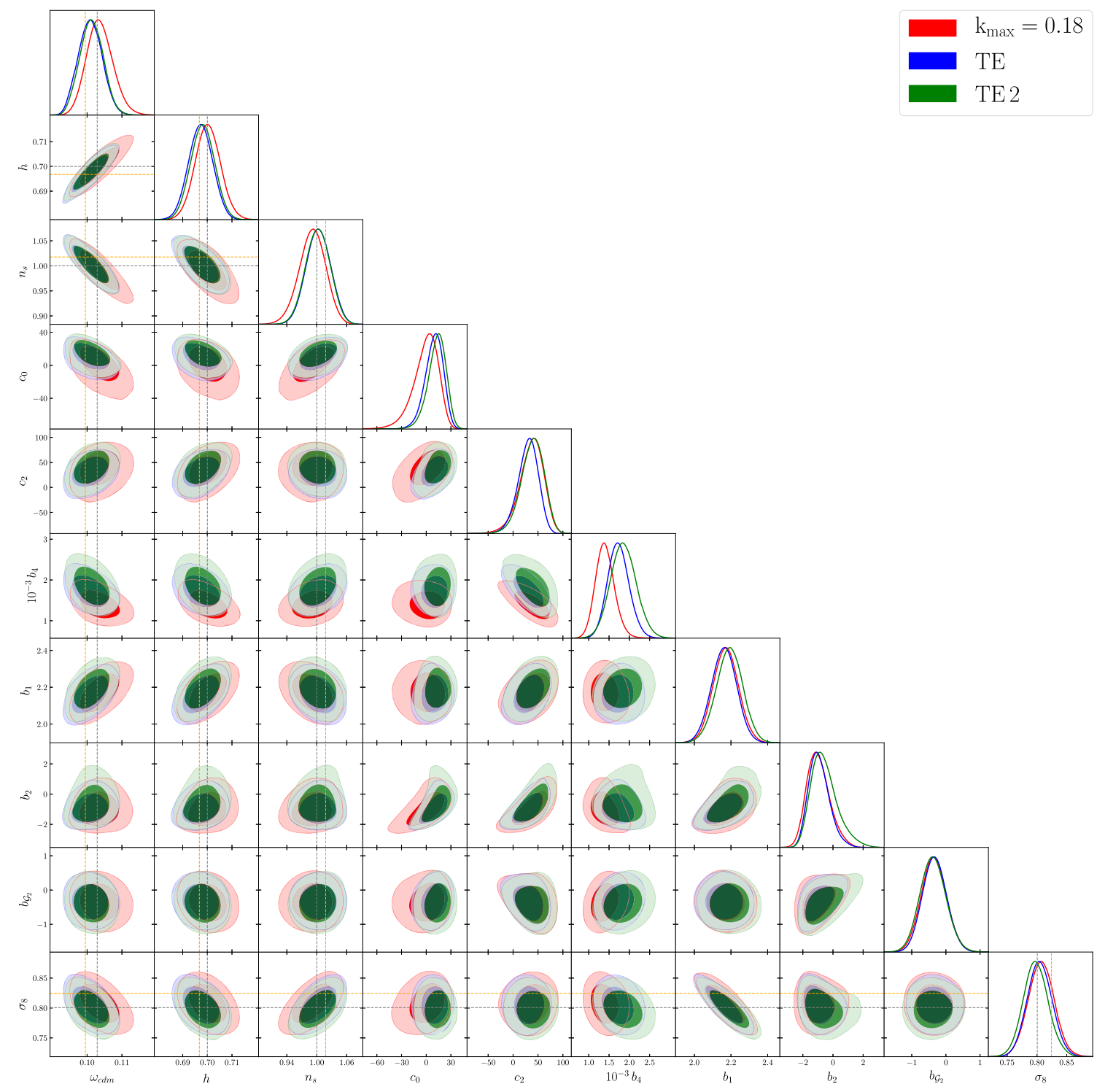

FIG. 21. Triangle plot for the cosmological and nuisance parameters measured from the redshift space galaxy power spectrum of the Las Damas simulations at $z=0.342$ for the $k_{\max }$ analysis and two choices of the fiducial cosmology in the TE approach. $c_{0}, c_{2}, b_{4}$ are quoted in units $\left[h^{-1} \mathrm{Mpc}\right]^{2},\left[h^{-1} \mathrm{Mpc}\right]^{2},\left[h^{-1} \mathrm{Mpc}\right]^{4}$, respectively. Crossing of the dashed orange lines outlines the fiducial cosmological parameters used in the TE 2 analysis.

[1] M. M. Ivanov, M. Simonović, and M. Zaldarriaga, Cosmological parameters from the BOSS galaxy power spectrum, J. Cosmol. Astropart. Phys. 05 (2020) 042.

[2] T. Colas, G. D'amico, L. Senatore, P. Zhang, and F. Beutler, Efficient cosmological analysis of the SDSS/BOSS data from the effective field theory of large-scale structure, J. Cosmol. Astropart. Phys. 06 (2020) 001.

[3] T. Tröster et al., Cosmology from large-scale structure: Constraining $\Lambda \mathrm{CDM}$ with BOSS, Astron. Astrophys. 633, L10 (2020).
[4] M. M. Ivanov, M. Simonović, and M. Zaldarriaga, Cosmological parameters and neutrino masses from the final planck and full-shape BOSS data, Phys. Rev. D 101, 083504 (2020).

[5] O. H. Philcox, M. M. Ivanov, M. Simonović, and M. Zaldarriaga, Combining full-shape and BAO analyses of galaxy power spectra: A 1.6CMB-independent constraint on H0, J. Cosmol. Astropart. Phys. 05 (2020) 032.

[6] O. H. Philcox, B. D. Sherwin, G. S. Farren, and E. J. Baxter, Determining the hubble constant without the sound horizon: 
Measurements from galaxy surveys, Phys. Rev. D (to be published).

[7] A. Chudaykin, K. Dolgikh, and M. M. Ivanov, Constraints on the curvature of the Universe and dynamical dark energy from the Full-shape and BAO data, Phys. Rev. D 103, 023507 (2021).

[8] R. Laureijs et al. (EUCLID Collaboration), Euclid definition study report, arXiv:1110.3193.

[9] L. Amendola et al., Cosmology and fundamental physics with the Euclid satellite, Living Rev. Relativity 21, 2 (2018).

[10] A. Aghamousa et al. (DESI Collaboration), The DESI experiment Part I: Science, targeting, and survey design, arXiv:1611.00036.

[11] A. Chudaykin and M. M. Ivanov, Measuring neutrino masses with large-scale structure: Euclid forecast with controlled theoretical error, J. Cosmol. Astropart. Phys. 11 (2019) 034.

[12] B. Audren, J. Lesgourgues, S. Bird, M. G. Haehnelt, and M. Viel, Neutrino masses and cosmological parameters from a Euclid-like survey: Markov Chain Monte Carlo forecasts including theoretical errors, J. Cosmol. Astropart. Phys. 01 (2013) 026.

[13] V. Yankelevich and C. Porciani, Cosmological information in the redshift-space bispectrum, Mon. Not. R. Astron. Soc. 483, 2078 (2019).

[14] M. M. Ivanov, E. McDonough, J. C. Hill, M. Simonović, M. W. Toomey, S. Alexander, and Matias Zaldarriaga, Constraining early dark energy with large-scale structure, Phys. Rev. D 102, 103502 (2020).

[15] R. Scoccimarro, M. Zaldarriaga, and L. Hui, Power spectrum correlations induced by nonlinear clustering, Astrophys. J. 527, 1 (1999).

[16] D. Wadekar and R. Scoccimarro, The galaxy power spectrum multipoles covariance in perturbation theory, Phys. Rev. D 102, 123517 (2020).

[17] K. Yamamoto, M. Nakamichi, A. Kamino, B. A. Bassett, and H. Nishioka, A measurement of the quadrupole power spectrum in the clustering of the $2 \mathrm{dF}$ QSO survey, Publ. Astron. Soc. Jpn. 58, 93 (2006).

[18] J. N. Grieb, A. G. Sánchez, S. Salazar-Albornoz, and C. Dalla Vecchia, Gaussian covariance matrices for anisotropic galaxy clustering measurements, Mon. Not. R. Astron. Soc. 457, 1577 (2016).

[19] C. Blake, P. Carter, and J. Koda, Power spectrum multipoles on the curved sky: An application to the 6-degree field galaxy survey, Mon. Not. R. Astron. Soc. 479, 5168 (2018).

[20] Y. Li, S. Singh, B. Yu, Y. Feng, and U. Seljak, Disconnected covariance of 2-point functions in large-scale structure, J. Cosmol. Astropart. Phys. 01 (2019) 016.

[21] F.-S. Kitaura et al., The clustering of galaxies in the SDSS-III baryon oscillation spectroscopic survey: mock galaxy catalogues for the BOSS Final Data Release, Mon. Not. R. Astron. Soc. 456, 4156 (2016).

[22] C. Zhao et al., The completed SDSS-IV extended baryon oscillation spectroscopic survey: One thousand multi-tracer mock catalogues with redshift evolution and systematics for galaxies and quasars of the final data release, arXiv: 2007.08997.

[23] S. Lin et al., The completed SDSS-IV extended baryon oscillation spectroscopic survey: GLAM-QPM mock galaxy catalogs for the emission line galaxy sample, Mon. Not. R. Astron. Soc. 498, 5251 (2020).

[24] J. Hartlap, P. Simon, and P. Schneider, Why your model parameter confidences might be too optimistic: Unbiased estimation of the inverse covariance matrix, Astron. Astrophys. 464, 399 (2007).

[25] W. J. Percival et al., The clustering of galaxies in the SDSSIII baryon oscillation spectroscopic survey: Including covariance matrix errors, Mon. Not. R. Astron. Soc. 439, 2531 (2014).

[26] E. Sellentin and A. F. Heavens, Parameter inference with estimated covariance matrices, Mon. Not. R. Astron. Soc. 456, L132 (2016).

[27] O. H. Philcox, M. M. Ivanov, M. Zaldarriaga, M. Simonovic, and M. Schmittfull, Fewer mocks and less noise: Reducing the dimensionality of cosmological observables with subspace projections, Phys. Rev. D (to be published).

[28] T. Baldauf, M. Mirbabayi, M. Simonović, and M. Zaldarriaga, LSS constraints with controlled theoretical uncertainties, arXiv:1602.00674.

[29] T. Steele and T. Baldauf, Precise calibration of the one-loop bispectrum in the effective field theory of large scale structure, Phys. Rev. D 103, 023520 (2021).

[30] G. D'Amico, J. Gleyzes, N. Kokron, D. Markovic, L. Senatore, P. Zhang, Florian Beutler, and Héctor Gil-Marín, The cosmological analysis of the SDSS/BOSS data from the effective field theory of large-scale structure, J. Cosmol. Astropart. Phys. 05 (2020) 005.

[31] T. Nishimichi, G. D’Amico, M. M. Ivanov, L. Senatore, M. Simonovic, M. Takada, Matias Zaldarriaga, and Pierre Zhang, Blinded challenge for precision cosmology with large-scale structure: Results from effective field theory for the redshift-space galaxy power spectrum, Phys. Rev. D 102, 123541 (2020).

[32] G. Rossi et al., The completed SDSS-IV extended baryon oscillation spectroscopic survey: N-body Mock challenge for galaxy clustering measurements, arXiv:2007.09002.

[33] S. Alam et al., The completed SDSS-IV extended baryon oscillation spectroscopic survey: N-body Mock challenge for the eBOSS emission line galaxy sample, arXiv:2007 .09004 .

[34] G. D'Amico, L. Senatore, and P. Zhang, Limits on $w \mathrm{CDM}$ from the EFTofLSS with the PyBird code, J. Cosmol. Astropart. Phys. 01 (2021) 006.

[35] L. Senatore and M. Zaldarriaga, The IR-resummed effective field theory of large scale structures, J. Cosmol. Astropart. Phys. 02 (2015) 013.

[36] T. Baldauf, M. Mirbabayi, M. Simonović, and M. Zaldarriaga, Equivalence principle and the baryon acoustic peak, Phys. Rev. D 92, 043514 (2015).

[37] Z. Vlah, U. Seljak, M. Y. Chu, and Y. Feng, Perturbation theory, effective field theory, and oscillations in the power spectrum, J. Cosmol. Astropart. Phys. 03 (2016) 057.

[38] D. Blas, M. Garny, M. M. Ivanov, and S. Sibiryakov, Timesliced perturbation theory II: Baryon acoustic oscillations and infrared resummation, J. Cosmol. Astropart. Phys. 07 (2016) 028.

[39] L. Senatore and G. Trevisan, On the IR-Resummation in the EFTofLSS, J. Cosmol. Astropart. Phys. 05 (2018) 019. 
[40] M. M. Ivanov and S. Sibiryakov, Infrared resummation for biased tracers in redshift space, J. Cosmol. Astropart. Phys. 07 (2018) 053.

[41] S.-F. Chen, Z. Vlah, and M. White, Consistent modeling of velocity statistics and redshift-space distortions in one-loop perturbation theory, J. Cosmol. Astropart. Phys. 07 (2020) 062.

[42] A. Vasudevan, M. M. Ivanov, S. Sibiryakov, and J. Lesgourgues, Time-sliced perturbation theory with primordial non-Gaussianity and effects of large bulk flows on inflationary oscillating features, J. Cosmol. Astropart. Phys. 09 (2019) 037.

[43] S.-F. Chen, Z. Vlah, and M. White, Modeling features in the redshift-space halo power spectrum with perturbation theory, J. Cosmol. Astropart. Phys. 11 (2020) 035.

[44] A. Chudaykin, M. M. Ivanov, and M. Simonović, CLASSPT: Non-linear perturbation theory extension of the Boltzmann code CLASS, Phys. Rev. D 102, 063533 (2020).

[45] M. Tegmark, Measuring Cosmological Parameters with Galaxy Surveys, Phys. Rev. Lett. 79, 3806 (1997).

[46] M. Tegmark, How to measure CMB power spectra without losing information, Phys. Rev. D 55, 5895 (1997).

[47] D. Wadekar, M. M. Ivanov, and R. Scoccimarro, Cosmological constraints from BOSS with analytic covariance matrices, Phys. Rev. D 102, 123521 (2020).

[48] D. Blas, M. Garny, M. M. Ivanov, and S. Sibiryakov, Time-sliced perturbation theory for large scale structure I: General formalism, J. Cosmol. Astropart. Phys. 07 (2016) 052.

[49] D. Baumann, A. Nicolis, L. Senatore, and M. Zaldarriaga, Cosmological non-linearities as an effective fluid, J. Cosmol. Astropart. Phys. 07 (2012) 051.

[50] J. J. M. Carrasco, M. P. Hertzberg, and L. Senatore, The effective field theory of cosmological large scale structures, J. High Energy Phys. 09 (2012) 082.

[51] A. Perko, L. Senatore, E. Jennings, and R. H. Wechsler, Biased tracers in redshift space in the EFT of large-scale structure, arXiv:1610.09321.

[52] C. McBride, A. Berlind, R. Scoccimarro, R. Wechsler, M. Busha, J. Gardner et al., LasDamas Mock Galaxy Catalogs for SDSS, Bull. Am. Astron. Soc. 41, 253 (2009), https://ui .adsabs.harvard.edu/abs/2009AAS...21342506M/abstract.

[53] M. M. Ivanov, Y. Ali-Haïmoud, and J. Lesgourgues, H0 tension or T0 tension?, Phys. Rev. D 102, 063515 (2020).

[54] http://lss.phy.vanderbilt.edu/lasdamas/overview.html

[55] S. Alam et al. (BOSS Collaboration), The clustering of galaxies in the completed SDSS-III baryon oscillation spectroscopic survey: Cosmological analysis of the DR12 galaxy sample, Mon. Not. R. Astron. Soc. 470, 2617 (2017).

[56] M. Simonović, T. Baldauf, M. Zaldarriaga, J. J. Carrasco, and J. A. Kollmeier, Cosmological perturbation theory using the FFTLog: Formalism and connection to QFT loop integrals, J. Cosmol. Astropart. Phys. 04 (2018) 030.

[57] B. Audren, J. Lesgourgues, K. Benabed, and S. Prunet, Conservative constraints on early cosmology: An illustration of the MontePython cosmological parameter inference code, J. Cosmol. Astropart. Phys. 02 (2013) 001.
[58] T. Brinckmann and J. Lesgourgues, MontePython 3: Boosted MCMC sampler and other features, Phys. Dark Universe 24, 100260 (2019).

[59] A. Lewis, GetDist: A Python package for analysing Monte Carlo samples, arXiv:1910.13970.

[60] N. Kaiser, Clustering in real space and in redshift space, Mon. Not. R. Astron. Soc. 227, 1 (1987).

[61] F. Bernardeau, S. Colombi, E. Gaztanaga, and R. Scoccimarro, Large scale structure of the universe and cosmological perturbation theory, Phys. Rep. 367, 1 (2002).

[62] T. Baldauf, L. Mercolli, and M. Zaldarriaga, Effective field theory of large scale structure at two loops: The apparent scale dependence of the speed of sound, Phys. Rev. D 92, 123007 (2015).

[63] J. C. Jackson, Fingers of God: A critique of Rees' theory of primoridal gravitational radiation, Mon. Not. R. Astron. Soc. 156, 1P (1972).

[64] L. Senatore and M. Zaldarriaga, Redshift space distortions in the effective field theory of large scale structures, arXiv:1409.1225.

[65] M. Lewandowski, L. Senatore, F. Prada, C. Zhao, and C.-H. Chuang, EFT of large scale structures in redshift space, Phys. Rev. D 97, 063526 (2018).

[66] R. Neveux et al., The completed SDSS-IV extended baryon oscillation spectroscopic survey: BAO and RSD measurements from the anisotropic power spectrum of the Quasar sample between redshift 0.8 and 2.2, Mon. Not. R. Astron. Soc. 499, 210 (2020).

[67] A. Smith et al., The completed SDSS-IV extended baryon oscillation spectroscopic survey: N-body Mock challenge for the Quasar sample, Mon. Not. R. Astron. Soc. 499, 269 (2020).

[68] F. Beutler et al. (BOSS Collaboration), The clustering of galaxies in the completed SDSS-III baryon oscillation spectroscopic survey: Anisotropic galaxy clustering in Fourier-space, Mon. Not. R. Astron. Soc. 466, 2242 (2017).

[69] T. Baldauf, U.S. Seljak, R. E. Smith, N. Hamaus, and V. Desjacques, Halo stochasticity from exclusion and nonlinear clustering, Phys. Rev. D 88, 083507 (2013).

[70] C. Hahn, R. Scoccimarro, M. R. Blanton, J. L. Tinker, and S. A. Rodríguez-Torres, The effect of fibre collisions on the galaxy power spectrum multipoles, Mon. Not. R. Astron. Soc. 467, 1940 (2017).

[71] M. Schmittfull, M. Simonović, V. Assassi, and M. Zaldarriaga, Modeling biased tracers at the field level, Phys. Rev. D 100, 043514 (2019).

[72] V. Desjacques, D. Jeong, and F. Schmidt, Large-scale galaxy bias, Phys. Rep. 733, 1 (2018).

[73] M. M. Abidi and T. Baldauf, Cubic Halo bias in Eulerian and Lagrangian space, J. Cosmol. Astropart. Phys. 07 (2018) 029.

[74] A. de Mattia et al., The completed SDSS-IV extended baryon oscillation spectroscopic survey: Measurement of the BAO and growth rate of structure of the emission line galaxy sample from the anisotropic power spectrum between redshift 0.6 and 1.1, arXiv:2007.09008.

[75] S. Pandey et al. (Dark Energy Survey Collaboration), Perturbation theory for modeling galaxy bias: Validation 
with simulations of the dark energy survey, Phys. Rev. D 102, 123522 (2020).

[76] http://hlit.jinr.ru

[77] D. Blas, M. Garny, and T. Konstandin, On the non-linear scale of cosmological perturbation theory, J. Cosmol. Astropart. Phys. 09 (2013) 024.
[78] D. Blas, M. Garny, and T. Konstandin, Cosmological perturbation theory at three-loop order, J. Cosmol. Astropart. Phys. 01 (2014) 010.

[79] S. Foreman, H. Perrier, and L. Senatore, Precision comparison of the power spectrum in the EFTofLSS with simulations, J. Cosmol. Astropart. Phys. 05 (2016) 027. 